\title{
Efficient and Unconditional Energy Stable Schemes for the Micropolar Navier-Stokes Equations
}

\author{
Jie Shen ${ }^{1}$ and Nan Zheng ${ }^{2, *}$ \\ ${ }^{1}$ Department of Mathematics, Purdue University, West Lafayette, IN 47907, USA. \\ 2 School of Mathematical Sciences and Fujian Provincial Key Laboratory on \\ Mathematical Modeling and High Performance Scientific Computing, Xiamen \\ University, Xiamen, Fujian, 361005, China.
}

Received 31 January 2021; Accepted 14 June 2021

\begin{abstract}
We develop in this paper efficient numerical schemes for solving the micropolar Navier-Stokes equations by combining the SAV approach and pressure-correction method. Our first- and second-order semi-discrete schemes enjoy remarkable properties such as (i) unconditional energy stable with a modified energy, and (ii) only a sequence of decoupled linear equations with constant coefficients need to be solved at each time step. We also construct fully discrete versions of these schemes with a special spectral discretization which preserve the essential properties of the semi-discrete schemes. Numerical experiments are presented to validate the proposed schemes.
\end{abstract}

AMS subject classifications: 65M12, 65M70, 35Q30, 76A05

Key words: Micropolar Navier-Stokes, pressure-correction, scalar auxiliary variable, energy stability.

\section{Introduction}

We consider in this paper the so-called micropolar Navier-Stokes equations [16]:

$$
\left\{\begin{array}{l}
\mathbf{u}_{t}+\mathbf{u} \cdot \nabla \mathbf{u}-v_{0} \Delta \mathbf{u}+\nabla p=2 v_{r} \nabla \times \mathbf{w}+\mathbf{f}, \\
\nabla \cdot \mathbf{u}=0, \\
j \mathbf{w}_{t}+j \mathbf{u} \cdot \nabla \mathbf{w}-c_{1} \Delta \mathbf{w}-c_{2} \nabla \nabla \cdot \mathbf{w}+4 v_{r} \mathbf{w}=2 v_{r} \nabla \times \mathbf{u}+\mathbf{g},
\end{array}\right.
$$

where $\mathbf{u}$ is the linear velocity vector, $p$ the pressure and $\mathbf{w}$ is the non-divergence free micro-rotation field (angular velocity of the rotation of particles of the fluid). The functions $\mathbf{f}(\mathbf{x})$ and $\mathbf{g}(\mathbf{x})$ denote external sources of linear and angular momentum, respectively [13], which depend upon external fields explicitly. The material constants $j, v_{0}, v_{r}$, $c_{1}$ and $c_{2}$ are all positive.

${ }^{*}$ Corresponding author. Email addresses: shen7@purdue.edu (J. Shen), znan2017@163.com (N. Zheng) 
This system is supplemented with the following initial and boundary conditions:

$$
\begin{aligned}
& \left.\mathbf{u}\right|_{t=0}=u_{0},\left.\quad \mathbf{u}\right|_{\partial \Omega}=0, \\
& \left.\mathbf{w}\right|_{t=0}=w_{0},\left.\quad \mathbf{w}\right|_{\partial \Omega}=0 .
\end{aligned}
$$

When the micro-rotation effect is ignored (i.e., $\mathbf{w}=0)$ ), (1.1) reduce to the celebrated Navier-Stokes equations. The kinematic Newtonian viscosity $v_{r}$ is essential for the flow of micropolar fluids, so that the velocity and the micro-rotation are coupled and the global motion is affected by the micro-rotations. The micropolar fluid is less prone to instability than the classical fluid $[5,7,22]$, and this model can describe some polymeric fluids and fluids containing certain additives in narrow films [6].

Mathematical properties of the micropolar Navier-Stokes equations have been studied in $[8,13,18,20]$. In particular, the global existence of weak solutions and the local existence and uniqueness of strong solutions are all well understood. From a numerical point of view, there are some attempts in designing numerical schemes for the micropolar Navier-Stokes equations. For instance, a penalty projection method is proposed and suboptimal error estimates are proved in [17]; a semi-implicit fully discrete scheme is presented in [15] which requires to solve a saddle point problem for velocity and pressure at each time step; in a related work [21], a fractional time stepping technique is proposed to decouple the computation of pressure and velocity. The nonlinear terms in these work are treated either implicitly or semi-implicitly, so that a coupled linear or nonlinear system with variable coefficients has to be solved at each time step.

Recently, the so called scalar auxiliary variable (SAV) approach [24] is introduced for gradient flows. The SAV approach introduces an auxiliary variable and treat all nonlinear terms explicitly, making it possible to construct linear, decoupled, unconditionally energy stable schemes which only require solving linear systems with constant coefficients at each time step. The SAV approach has also been successfully applied to solve Navier-Stokes and related dissipative equations which are not gradient flows $[11,12]$. In this paper, we combine the SAV approach with the pressure-correction method (see, e.g., [10]) to construct efficient numerical schemes for the micropolar Navier Stokes equations. More precisely, the semi-discrete schemes we construct using the SAV approach enjoy the following remarkable properties: (i) unconditionally energy stable with a modified energy; and (ii) at each time step, only a sequence of Poisson type equations need to be solved.

Unlike in the case of gradient flows for which any consistent Galerkin type spatial discretization will preserve the nice properties of the semi-discrete SAV schemes, special care has to be taken to choose compatible spatial discretization for pressure and velocity, which is different from the usual inf-sup compatible discretization in a coupled approach. We constructed fully discrete schemes with Galerkin spectral-discretization in space that full preserve the two essential properties of the semi-discrete SAV schemes, and present several numerical experiments to validate our results.

The reminder of the paper is organized as follows. In Section 2, we construct the semi-discrete first- and second-order SAV schemes with pressure-correction to decouple 
the pressure from the velocity. In Section 3, we prove that both the first- and second-order schemes constructed in the last section are unconditionally energy stable with a modified energy. In Section 4, we construct a fully discrete scheme and prove its stability. Some numerical experiments are presented in Section 5 followed by some concluding remarks in Section 6.

\section{SAV scheme for the micropolar NSEs}

We start with some notations and results that will be subsequently used.

We consider the system (1.1) on a finite time interval $[0, T]$ and in an open, bounded, connected domain $\Omega \subset \mathbb{R}^{d}$ with smooth boundary $\partial \Omega$. Let us introduce the standard Sobolev spaces $H^{m}(\Omega)$ whose norm are denoted by $\|\cdot\|_{m}$. In particular, the norm and inner product of $L^{2}(\Omega)$ are denoted by $\|\cdot\|$ and $(\cdot, \cdot)$, respectively.

We denote

$$
\mathbf{H}=\left\{\mathbf{u} \in \mathbf{L}^{2}(\Omega)|\operatorname{div} \mathbf{u}=0, \mathbf{u} \cdot \mathbf{n}|_{\Gamma}=0\right\}, \quad \mathbf{V}=\mathbf{H}_{0}^{1}(\Omega) \cap \mathbf{H} .
$$

Recall the following identity

$$
\|\nabla \mathbf{u}\|^{2}=\|\nabla \times \mathbf{u}\|^{2}+\|\nabla \cdot \mathbf{u}\|^{2}, \quad \forall \mathbf{u} \in \mathbf{H}_{0}^{1}(\Omega) .
$$

Denote

$$
b(\mathbf{u}, \mathbf{v}, \mathbf{w})=\int_{\Omega}(\mathbf{u} \cdot \nabla) \mathbf{v} \cdot \mathbf{w} d \mathbf{x},
$$

which satisfies the following skew-symmetric property

$$
b(\mathbf{u}, \mathbf{v}, \mathbf{w})=-b(\mathbf{u}, \mathbf{w}, \mathbf{v}), \quad \forall \mathbf{u} \in \mathbf{H}, \mathbf{v}, \mathbf{w} \in \mathbf{H}_{0}^{1}(\Omega) .
$$

The micropolar NSEs (1.1) satisfy a dissipative energy law. Indeed, taking the inner product of the first equation in (1.1) with $\mathbf{u}$ and the last equation in (1.1) with $\mathbf{w}$, in the absence of the external force $\mathbf{f}$ and $\mathbf{g}$, we obtain immediately the energy dissipation law:

$$
\begin{aligned}
& \frac{d}{d t}\left(\frac{1}{2}\|\mathbf{u}\|^{2}+\frac{j}{2}\|\mathbf{w}\|^{2}\right) \\
= & -\left(v_{0}\|\nabla \mathbf{u}\|^{2}+c_{1}\|\nabla \mathbf{w}\|^{2}+c_{2}\|\nabla \cdot \mathbf{w}\|^{2}+4 v_{r}\|\nabla \mathbf{w}\|^{2}\right) .
\end{aligned}
$$

Given a constant $C_{0}>0$, we introduce a SAV $R(t)$ defined by

$$
R^{2}(t)=\int_{\Omega} \frac{1}{4}\left(|\mathbf{u}(t)|^{2}+j|\mathbf{w}(t)|^{2}\right) d \mathbf{x}+C_{0}:=E(t),
$$

where $E(t)-C_{0}$ and $R^{2}(t)-C_{0}$ is half of the total energy $\frac{1}{2}\|\mathbf{u}\|^{2}+\frac{j}{2}\|\mathbf{w}\|^{2}$. 
Taking the time derivative on both sides and using (2.4), we have

$$
\begin{aligned}
2 R R_{t}= & \int_{\Omega} \frac{1}{2}\left(\mathbf{u u}_{t}+j \mathbf{w} \mathbf{w}_{t}\right) d \mathbf{x} \\
= & \int_{\Omega} \frac{1}{2}\left(\mathbf{u} \mathbf{u}_{t}+j \mathbf{w} \mathbf{w}_{t}\right)+\mathbf{u} \cdot \nabla \mathbf{u} \cdot \mathbf{u}+j \mathbf{u} \cdot \nabla \mathbf{w} \cdot \mathbf{w} \\
& \quad-2 v_{r}(\mathbf{u} \nabla \times \mathbf{w}+\mathbf{w} \nabla \times \mathbf{u}) d \mathbf{x} .
\end{aligned}
$$

We can then reformulate (1.1) into the following expanded system:

$$
\begin{aligned}
& \mathbf{u}_{t}+\frac{R(t)}{\sqrt{E(t)}} \mathbf{u} \cdot \nabla \mathbf{u}-v_{0} \triangle \mathbf{u}+\nabla p=2 v_{r} \frac{R(t)}{\sqrt{E(t)}} \nabla \times \mathbf{w}+\mathbf{f}, \\
& \nabla \cdot \mathbf{u}=0, \\
& j \mathbf{w}_{t}+j \frac{R(t)}{\sqrt{E(t)}} \mathbf{u} \cdot \nabla \mathbf{w}-c_{1} \Delta \mathbf{w}-c_{2} \nabla \nabla \cdot \mathbf{w}+4 v_{r} \mathbf{w}=2 v_{r} \frac{R(t)}{\sqrt{E(t)}} \nabla \times \mathbf{u}+\mathbf{g}, \\
& 2 R R_{t}=\int_{\Omega} \frac{1}{2}\left(\mathbf{u} \mathbf{u}_{t}+j \mathbf{w} \mathbf{w}_{t}\right)+\frac{R(t)}{\sqrt{E(t)}}(\mathbf{u} \cdot \nabla \mathbf{u} \cdot \mathbf{u}+j \mathbf{u} \cdot \nabla \mathbf{w} \cdot \mathbf{w} \\
& \left.-2 v_{r} \mathbf{u} \nabla \times \mathbf{w}-2 v_{r} \mathbf{w} \nabla \times \mathbf{u}\right) d \mathbf{x} .
\end{aligned}
$$

If $R(0)=\sqrt{E(0)}$, we find that a solution to the above system is $R(t)=\sqrt{E(t)}$ with $(\mathbf{u}, \mathbf{w})$ being the solution of (1.1). Instead of solving (1.1), we shall solve the expanded system (2.8).

\subsection{The first-order SAV pressure-correction scheme}

In order to construct a totally decoupled unconditional energy stable scheme, we combine the SAV approach for Navier-Stokes equations [12] to deal with nonlinear terms and the standard pressure-correction method [10] to decouple the pressure from the velocity.

Scheme I. Assuming $\tilde{\mathbf{u}}^{n}, \mathbf{u}^{n}, \mathbf{w}^{n}$ and $p^{n}$ are known, we update $\tilde{\mathbf{u}}^{n+1}, \mathbf{u}^{n+1}, \mathbf{w}^{n+1}$ and $p^{n+1}$ as follows:

$$
\begin{gathered}
\frac{\tilde{\mathbf{u}}^{n+1}-\mathbf{u}^{n}}{\Delta t}+\frac{R^{n+1}}{\sqrt{E^{n}}} \mathbf{u}^{n} \cdot \nabla \mathbf{u}^{n}-v_{0} \triangle \tilde{\mathbf{u}}^{n+1}+\nabla p^{n} \\
=2 v_{r} \frac{R^{n+1}}{\sqrt{E^{n}}} \nabla \times \mathbf{w}^{n}+\mathbf{f}^{n+1},\left.\quad \tilde{\mathbf{u}}^{n+1}\right|_{\partial \Omega}=0 \\
\frac{\mathbf{u}^{n+1}-\tilde{\mathbf{u}}^{n+1}}{\Delta t}+\nabla\left(p^{n+1}-p^{n}\right)=0, \\
\nabla \cdot \mathbf{u}^{n+1}=0,\left.\quad \mathbf{u}^{n+1} \cdot n\right|_{\partial \Omega}=0
\end{gathered}
$$




$$
\begin{aligned}
j \frac{\mathbf{w}^{n+1}-\mathbf{w}^{n}}{\Delta t}+j \frac{R^{n+1}}{\sqrt{E^{n}}} \mathbf{u}^{n} \cdot \nabla \mathbf{w}^{n}-c_{1} \triangle \mathbf{w}^{n+1} \\
-c_{2} \nabla \nabla \cdot \mathbf{w}^{n+1}+4 v_{r} \mathbf{w}^{n+1}=2 v_{r} \frac{R^{n+1}}{\sqrt{E^{n}}} \nabla \times \tilde{\mathbf{u}}^{n}+\mathbf{g}^{n+1} ; \\
2 R^{n+1} \frac{R^{n+1}-R^{n}}{\Delta t}=\int_{\Omega} \frac{1}{2}\left(\mathbf{u}^{n+1} \frac{\mathbf{u}^{n+1}-\mathbf{u}^{n}}{\Delta t}+j \mathbf{w}^{n+1} \frac{\mathbf{w}^{n+1}-\mathbf{w}^{n}}{\Delta t}\right) \\
+\frac{R^{n+1}}{\sqrt{E^{n}}}\left(\mathbf{u}^{n} \cdot \nabla \mathbf{u}^{n} \cdot \tilde{\mathbf{u}}^{n+1}+j \mathbf{u}^{n} \cdot \nabla \mathbf{w}^{n} \cdot \mathbf{w}^{n+1}\right. \\
\left.-2 v_{r} \tilde{\mathbf{u}}^{n+1} \nabla \times \mathbf{w}^{n}-2 v_{r} \mathbf{w}^{n+1} \nabla \times \tilde{\mathbf{u}}^{n}\right) d \mathbf{x} ;
\end{aligned}
$$

where

$$
E^{n}=\frac{1}{4}\left(\left\|\mathbf{u}^{n}\right\|^{2}+j\left\|\mathbf{w}^{n}\right\|^{2}\right)+C_{0} .
$$

The above scheme is weakly coupled together by $R^{n+1}$, and can be efficiently implemented as follows:

Setting $\xi^{n+1}=\frac{R^{n+1}}{\sqrt{E^{n}}}$ and

$$
\begin{aligned}
& \tilde{\mathbf{u}}^{n+1}=\tilde{\mathbf{u}}_{1}^{n+1}+\xi^{n+1} \tilde{\mathbf{u}}_{2}^{n+1}, \quad \mathbf{u}^{n+1}=\mathbf{u}_{1}^{n+1}+\xi^{n+1} \mathbf{u}_{2}^{n+1}, \\
& \mathbf{w}^{n+1}=\mathbf{w}_{1}^{n+1}+\xi^{n+1} \mathbf{w}_{2}^{n+1}, \quad p^{n+1}=p_{1}^{n+1}+\xi^{n+1} p_{2}^{n+1},
\end{aligned}
$$

in (2.9a)-(2.9c), and collect the terms with or without $\xi^{n+1}$ respectively, we obtain the following decoupled equations for $\left(\tilde{\mathbf{u}}_{i}^{n+1}, \mathbf{u}_{i}^{n+1}, \mathbf{w}_{i}^{n+1}, p_{i}^{n+1}\right), i=1,2$ :

$$
\begin{aligned}
& \frac{\tilde{\mathbf{u}}_{1}^{n+1}-\mathbf{u}^{n}}{\Delta t}-v_{0} \triangle \tilde{\mathbf{u}}_{1}^{n+1}+\nabla p_{1}^{n}=\mathbf{f}^{n+1},\left.\quad \tilde{\mathbf{u}}_{1}^{n+1}\right|_{\partial \Omega}=0, \\
& \triangle\left(p_{1}^{n+1}-p_{1}^{n}\right)=\frac{1}{\Delta t} \nabla \cdot \tilde{\mathbf{u}}_{1}^{n+1},\left.\quad \frac{\partial\left(p_{1}^{n+1}-p_{1}^{n}\right)}{\partial n}\right|_{\partial \Omega}=0, \\
& \mathbf{u}_{1}^{n+1}=\tilde{\mathbf{u}}_{1}^{n+1}-\Delta t \nabla\left(p_{1}^{n+1}-p_{1}^{n}\right), \\
& j \frac{\mathbf{w}_{1}^{n+1}-\mathbf{w}^{n}}{\Delta t}-c_{1} \triangle \mathbf{w}_{1}^{n+1}-c_{2} \nabla \nabla \cdot \mathbf{w}_{1}^{n+1} \\
& +4 v_{r} \mathbf{w}_{1}^{n+1}=\mathbf{g}^{n+1},\left.\quad \mathbf{w}_{1}^{n+1}\right|_{\partial \Omega}=0,
\end{aligned}
$$

and

$$
\begin{aligned}
& \frac{\tilde{\mathbf{u}}_{2}^{n+1}}{\Delta t}+\mathbf{u}^{n} \cdot \nabla \mathbf{u}^{n}-v_{0} \triangle \tilde{\mathbf{u}}_{2}^{n+1}+\nabla p_{2}^{n}=2 v_{r} \nabla \times \mathbf{w}^{n},\left.\quad \tilde{\mathbf{u}}_{2}^{n+1}\right|_{\partial \Omega}=0, \\
& \begin{aligned}
\triangle\left(p_{2}^{n+1}-p_{2}^{n}\right) & =\frac{1}{\Delta t} \nabla \cdot \tilde{\mathbf{u}}_{2}^{n+1},\left.\quad \frac{\partial\left(p_{2}^{n+1}-p_{2}^{n}\right)}{\partial n}\right|_{\partial \Omega}=0, \\
\mathbf{u}_{2}^{n+1} & =\tilde{\mathbf{u}}_{2}^{n+1}-\Delta t \nabla\left(p_{2}^{n+1}-p_{2}^{n}\right),
\end{aligned}
\end{aligned}
$$




$$
\begin{gathered}
j \frac{\mathbf{w}_{2}^{n+1}}{\Delta t}+j \mathbf{u}^{n} \cdot \nabla \mathbf{w}^{n}-c_{1} \Delta \mathbf{w}_{2}^{n+1}-c_{2} \nabla \nabla \cdot \mathbf{w}_{2}^{n+1} \\
\quad+4 v_{r} \mathbf{w}_{2}^{n+1}=2 v_{r} \nabla \times \tilde{\mathbf{u}}^{n},\left.\quad \mathbf{w}_{2}^{n+1}\right|_{\partial \Omega}=0 .
\end{gathered}
$$

Each of the above decoupled equations is a Poisson type equation so it can be efficiently solved. After we solve (2.12)-(2.13), we plug (2.11) into (2.9d), to obtain a nonlinear quadratic algebraic system for $R^{n+1}$ which can be solved directly using the quadratic formula or with a Newton iteration. Note that the time step may need to be sufficiently small for $(2.9 \mathrm{~d})$ to have a real positive solution.

To summarize, the algorithm consists of the following steps:

Step 1: Compute $\left(\tilde{\mathbf{u}}_{i}^{n+1}, i=1,2\right)$ from (2.12a) and (2.13a), then determine $\left(\mathbf{u}_{i}^{n+1}, p_{i}^{n+1}, i=\right.$ $1,2)$ from $(2.12 b)$ and $(2.13 b)$. Finally, obtain $\left(\mathbf{w}_{i}^{n+1}, i=1,2\right)$ from (2.12c) and (2.13c);

Step 2: Plug (2.11) into (2.9d), and solve $R^{n+1}$ by using a Newton iteration;

Step 3: Determine $\tilde{\mathbf{u}}^{n+1}, \mathbf{u}^{n+1}, p^{n+1}, \mathbf{w}^{n+1}$ from (2.11), goto the next time step.

\subsection{A second-order SAV rotational pressure-correction scheme}

We can construct a second-order scheme by combining the SAV approach with a rotational pressure-correction method [10]. To simplicity the notation, for any sequence $\left\{v^{n}\right\}$, we denote $\bar{v}^{n+1}=2 v^{n}-v^{n-1}$.

Scheme II. Assuming $\tilde{\mathbf{u}}^{n-1}, \tilde{\mathbf{u}}^{n}, \mathbf{u}^{n-1}, \mathbf{u}^{n}, \mathbf{w}^{n-1}, \mathbf{w}^{n}$ and $p^{n-1}, p^{n}$ are known, we update $\tilde{\mathbf{u}}^{n+1}, \mathbf{u}^{n+1}, \mathbf{w}^{n+1}$ and $p^{n+1}$ as follows:

$$
\begin{aligned}
& \frac{3 \tilde{\mathbf{u}}^{n+1}-4 \mathbf{u}^{n}+\mathbf{u}^{n-1}}{2 \Delta t}+\frac{R^{n+1}}{\sqrt{\bar{E}^{n+1}}} \overline{\mathbf{u}}^{n+1} \cdot \nabla \overline{\mathbf{u}}^{n+1} \\
& -v_{0} \Delta \tilde{\mathbf{u}}^{n+1}+\nabla p^{n}=2 v_{r} \frac{R^{n+1}}{\sqrt{\bar{E}^{n+1}}} \nabla \times \overline{\mathbf{w}}^{n+1}+\mathbf{f}^{n+1},\left.\quad \tilde{\mathbf{u}}^{n+1}\right|_{\partial \Omega}=0 ; \\
& \frac{3 \mathbf{u}^{n+1}-3 \tilde{\mathbf{u}}^{n+1}}{2 \Delta t}+\nabla\left(p^{n+1}-p^{n}+\chi v_{0} \nabla \tilde{\mathbf{u}}^{n+1}\right)=0, \\
& \nabla \cdot \mathbf{u}^{n+1}=0,\left.\quad \mathbf{u}^{n+1} \cdot n\right|_{\partial \Omega}=0 ; \\
& j \frac{3 \mathbf{w}^{n+1}-4 \mathbf{w}^{n}+\mathbf{w}^{n-1}}{2 \Delta t}+j \frac{R^{n+1}}{\sqrt{\bar{E}^{n+1}}} \overline{\mathbf{u}}^{n+1} \cdot \nabla \overline{\mathbf{w}}^{n+1}-c_{1} \Delta \mathbf{w}^{n+1} \\
& -c_{2} \nabla \nabla \cdot \mathbf{w}^{n+1}+4 v_{r} \mathbf{w}^{n+1}=2 v_{r} \frac{R^{n+1}}{\sqrt{\bar{E}^{n+1}}} \nabla \times \overline{\mathbf{u}}^{n+1}+\mathbf{g}^{n+1} ;
\end{aligned}
$$




$$
\begin{aligned}
2 R^{n+1} \frac{3 R^{n+1}-4 R^{n}+R^{n-1}}{2 \Delta t}= & \int_{\Omega} \frac{1}{2}\left(\mathbf{u}^{n+1} \frac{3 \mathbf{u}^{n+1}-4 \mathbf{u}^{n}+\mathbf{u}^{n-1}}{2 \Delta t}+j \mathbf{w}^{n+1} \frac{3 \mathbf{w}^{n+1}-4 \mathbf{w}^{n}+\mathbf{w}^{n-1}}{2 \Delta t}\right) \\
& +\frac{R^{n+1}}{\sqrt{\bar{E}^{n+1}}}\left(\overline{\mathbf{u}}^{n+1} \cdot \nabla \overline{\mathbf{u}}^{n+1} \cdot \tilde{\mathbf{u}}^{n+1}+j \overline{\mathbf{u}}^{n+1} \cdot \nabla \overline{\mathbf{w}}^{n+1} \cdot \mathbf{w}^{n+1}\right. \\
& \left.-2 v_{r} \tilde{\mathbf{u}}^{n+1} \nabla \times \overline{\mathbf{w}}^{n+1}-2 v_{r} \mathbf{w}^{n+1} \nabla \times \overline{\mathbf{u}}^{n+1}\right) d \mathbf{x} ;
\end{aligned}
$$

where $\bar{E}^{n+1}=2 E^{n}-E^{n-1}$ with $E^{k}$ defined in (2.10).

The above scheme can be efficiently implemented as the first-order scheme. More precisely, setting $\xi^{n+1}=\frac{R^{n+1}}{\sqrt{\bar{E}^{n+1}}}$ and

$$
\begin{aligned}
& \tilde{\mathbf{u}}^{n+1}=\tilde{\mathbf{u}}_{1}^{n+1}+\xi^{n+1} \tilde{\mathbf{u}}_{2}^{n+1}, \quad \mathbf{u}^{n+1}=\mathbf{u}_{1}^{n+1}+\xi^{n+1} \mathbf{u}_{2}^{n+1}, \\
& \mathbf{w}^{n+1}=\mathbf{w}_{1}^{n+1}+\xi^{n+1} \mathbf{w}_{2}^{n+1}, \quad p^{n+1}=p_{1}^{n+1}+\xi^{n+1} p_{2}^{n+1},
\end{aligned}
$$

in (2.14a)-(2.14c), and collect the terms with or without $\xi^{n+1}$ respectively, we obtain the following decoupled equations for $\left(\tilde{\mathbf{u}}_{i}^{n+1}, \mathbf{u}_{i}^{n+1}, \mathbf{w}_{i}^{n+1}, p_{i}^{n+1}\right), i=1,2$ :

$$
\begin{aligned}
& \frac{3 \tilde{\mathbf{u}}_{1}^{n+1}-4 \mathbf{u}^{n}+\mathbf{u}^{n-1}}{2 \Delta t}-v_{0} \triangle \tilde{\mathbf{u}}_{1}^{n+1}+\nabla p_{1}^{n}=\mathbf{f}^{n+1},\left.\quad \tilde{\mathbf{u}}_{1}^{n+1}\right|_{\partial \Omega}=0, \\
& \triangle \phi_{1}^{n+1}=\frac{3}{2 \Delta t} \nabla \cdot \tilde{\mathbf{u}}_{1}^{n+1},\left.\quad \frac{\partial \phi_{1}^{n+1}}{\partial n}\right|_{\partial \Omega}=0 \\
& \mathbf{u}_{1}^{n+1}=\tilde{\mathbf{u}}_{1}^{n+1}-\frac{2 \Delta t}{3} \nabla \phi_{1}^{n+1}, \quad \phi_{1}^{n+1}=p_{1}^{n+1}-p_{1}^{n}+\chi v_{0} \nabla \cdot \tilde{\mathbf{u}}_{1}^{n+1} ; \\
& j \frac{3 \mathbf{w}_{1}^{n+1}-4 \mathbf{w}^{n}+\mathbf{w}^{n-1}}{2 \Delta t}-c_{1} \triangle \mathbf{w}_{1}^{n+1}-c_{2} \nabla \nabla \cdot \mathbf{w}_{1}^{n+1}+4 v_{r} \mathbf{w}_{1}^{n+1}=\mathbf{g}^{n+1}, \\
& \left.\mathbf{w}_{1}^{n+1}\right|_{\partial \Omega}=0
\end{aligned}
$$

and

$$
\begin{aligned}
& \frac{3 \tilde{\mathbf{u}}_{2}^{n+1}}{2 \Delta t}+\overline{\mathbf{u}}^{n+1} \cdot \nabla \overline{\mathbf{u}}^{n+1}-v_{0} \triangle \tilde{\mathbf{u}}_{2}^{n+1}+\nabla p_{2}^{n}=2 v_{r} \nabla \times \overline{\mathbf{w}}^{n+1},\left.\quad \tilde{\mathbf{u}}_{2}^{n+1}\right|_{\partial \Omega}=0, \\
& \triangle \phi_{2}^{n+1}=\frac{3}{2 \Delta t} \nabla \cdot \tilde{\mathbf{u}}_{2}^{n+1},\left.\quad \frac{\partial \phi_{2}^{n+1}}{\partial n}\right|_{\partial \Omega}=0, \\
& \quad \mathbf{u}_{2}^{n+1}=\tilde{\mathbf{u}}_{2}^{n+1}-\frac{2 \Delta t}{3} \nabla \phi_{2}^{n+1}, \quad \phi_{2}^{n+1}=p_{2}^{n+1}-p_{2}^{n}+\chi v_{0} \nabla \cdot \tilde{\mathbf{u}}_{2}^{n+1}, \\
& j \frac{3 \mathbf{w}_{2}^{n+1}}{2 \Delta t}+j \overline{\mathbf{u}}^{n+1} \cdot \nabla \overline{\mathbf{w}}^{n+1}-c_{1} \triangle \mathbf{w}_{2}^{n+1}-c_{2} \nabla \nabla \cdot \mathbf{w}_{2}^{n+1}+4 v_{r} \mathbf{w}_{2}^{n+1}=2 v_{r} \nabla \times \overline{\mathbf{u}}^{n+1}, \\
& \left.\quad \mathbf{w}_{2}^{n+1}\right|_{\partial \Omega}=0 .
\end{aligned}
$$

After we solve (2.16)-(2.17), we plug (2.15) into (2.14d) to obtain a nonlinear algebraic equation for $R^{n+1}$ which can be solved with a Newton iteration. Hence, the computational procedure for the second-order scheme is essentially the same as the first-order scheme, only requiring solving a sequence of Poisson type equations plus a nonlinear algebraic equation at each time step. 


\section{Unconditional energy stability}

We prove in this sections that the two schemes presented in the last section are unconditionally energy stable.

We start with the first-order scheme.

Theorem 3.1. In the absence of the external force $f$ and $g$, the scheme (2.9) satisfies the following discrete energy law unconditionally:

$$
\mathcal{E}^{n+1}-\mathcal{E}^{n} \leq-\Delta t\left(v_{0}\left\|\nabla \tilde{\boldsymbol{u}}^{n+1}\right\|^{2}+c_{1}\left\|\nabla \boldsymbol{w}^{n+1}\right\|^{2}+c_{2}\left\|\nabla \cdot \boldsymbol{w}^{n+1}\right\|^{2}+4 v_{r}\left\|\boldsymbol{w}^{n+1}\right\|^{2}\right),
$$

where

$$
\mathcal{E}^{n+1}=\frac{1}{4}\left\|\boldsymbol{u}^{n+1}\right\|^{2}+\frac{j}{4}\left\|\boldsymbol{w}^{n+1}\right\|^{2}+\left|R^{n+1}\right|^{2}+\frac{\Delta t^{2}}{2}\left\|\nabla p^{n+1}\right\|^{2} .
$$

Proof. Take the inner product of (2.9a) with $\tilde{\mathbf{u}}^{n+1}$ and use the identity

$$
a(a-b)=\frac{1}{2} a^{2}-\frac{1}{2} b^{2}+\frac{1}{2}(a-b)^{2},
$$

we obtain

$$
\begin{gathered}
\frac{1}{2 \Delta t}\left(\left\|\tilde{\mathbf{u}}^{n+1}\right\|^{2}-\left\|\mathbf{u}^{n}\right\|^{2}\right)+\frac{1}{2 \Delta t}\left\|\tilde{\mathbf{u}}^{n+1}-\mathbf{u}^{n}\right\|^{2}+\frac{R^{n+1}}{\sqrt{E^{n+1}}}\left(\mathbf{u}^{n} \cdot \nabla \mathbf{u}^{n}, \tilde{\mathbf{u}}^{n+1}\right) \\
+v_{0}\left\|\nabla \tilde{\mathbf{u}}^{n+1}\right\|^{2}+\left(\nabla p^{n}, \tilde{\mathbf{u}}^{n+1}\right)=2 v_{r} \frac{R^{n+1}}{\sqrt{E^{n+1}}}\left(\nabla \times \mathbf{w}^{n}, \tilde{\mathbf{u}}^{n+1}\right) .
\end{gathered}
$$

Next, we rewrite $(2.9 b)$ as

$$
\mathbf{u}^{n+1}+\Delta t \nabla p^{n+1}=\tilde{\mathbf{u}}^{n+1}+\Delta t \nabla p^{n},
$$

and take the inner product of above with itself on both sides, notice that $\left(\nabla p^{k+1}, \mathbf{u}^{k+1}\right)=$ $-\left(p^{k+1}, \nabla \cdot \mathbf{u}^{k+1}\right)=0$, we get

$$
\left\|\mathbf{u}^{n+1}\right\|^{2}+\Delta t^{2}\left\|\nabla p^{n+1}\right\|^{2}=\left\|\tilde{\mathbf{u}}^{n+1}\right\|^{2}+2 \Delta t\left(\tilde{\mathbf{u}}^{n+1}, \nabla p^{n}\right)+\Delta t^{2}\left\|\nabla p^{n}\right\|^{2} .
$$

Summing up (3.4) and (3.6), we obtain

$$
\begin{aligned}
& \quad \frac{1}{2 \Delta t}\left(\left\|\mathbf{u}^{n+1}\right\|^{2}-\left\|\mathbf{u}^{n}\right\|^{2}\right)+\frac{1}{2 \Delta t}\left\|\tilde{\mathbf{u}}^{n+1}-\mathbf{u}^{n}\right\|^{2}+\frac{\Delta t}{2}\left\|\nabla p^{n+1}\right\|^{2}-\frac{\Delta t}{2}\left\|\nabla p^{n}\right\|^{2} \\
& +\frac{R^{n+1}}{\sqrt{E^{n+1}}}\left(\mathbf{u}^{n} \cdot \nabla \mathbf{u}^{n}, \tilde{\mathbf{u}}^{n+1}\right)+v_{0}\left\|\nabla \tilde{\mathbf{u}}^{n+1}\right\|^{2} \\
& =2 v_{r} \frac{R^{n+1}}{\sqrt{E^{n+1}}}\left(\nabla \times \mathbf{w}^{n}, \tilde{\mathbf{u}}^{n+1}\right) .
\end{aligned}
$$


Taking the inner product of $(2.9 \mathrm{c})$ with $\mathbf{w}^{n+1}$, we obtain

$$
\begin{aligned}
& \frac{j}{2 \Delta t}\left(\left\|\mathbf{w}^{n+1}\right\|^{2}-\left\|\mathbf{w}^{n}\right\|^{2}\right)+\frac{j}{2 \Delta t}\left\|\mathbf{w}^{n+1}-\mathbf{w}^{n}\right\|^{2}+j \frac{R^{n+1}}{\sqrt{E^{n+1}}}\left(\mathbf{u}^{n} \cdot \nabla \mathbf{w}^{n}, \mathbf{w}^{n+1}\right) \\
& +c_{1}\left\|\nabla \mathbf{w}^{n+1}\right\|^{2}+c_{2}\left\|\nabla \cdot \mathbf{w}^{n+1}\right\|^{2}+4 v_{r}\left\|\mathbf{w}^{n+1}\right\|^{2} \\
= & 2 v_{r} \frac{R^{n+1}}{\sqrt{E^{n+1}}}\left(\nabla \times \tilde{\mathbf{u}}^{n}, \mathbf{w}^{n+1}\right) .
\end{aligned}
$$

On the other hand, we derive from (2.9d) as

$$
\begin{aligned}
& \frac{1}{\Delta t}\left(\left|R^{n+1}\right|^{2}-\left|R^{n}\right|^{2}+\left|R^{n+1}-R^{n}\right|^{2}\right) \\
= & \frac{1}{4 \Delta t}\left(\left\|\mathbf{u}^{n+1}\right\|^{2}-\left\|\mathbf{u}^{n}\right\|^{2}\right)+\frac{1}{4 \Delta t}\left\|\mathbf{u}^{n+1}-\mathbf{u}^{n}\right\|^{2} \\
& +\frac{j}{4 \Delta t}\left(\left\|\mathbf{w}^{n+1}\right\|^{2}-\left\|\mathbf{w}^{n}\right\|^{2}\right)+\frac{j}{4 \Delta t}\left\|\mathbf{w}^{n+1}-\mathbf{w}^{n}\right\|^{2} \\
& +\int_{\Omega} \frac{R^{n+1}}{\sqrt{E^{n}}}\left(\mathbf{u}^{n} \cdot \nabla \mathbf{u}^{n} \cdot \tilde{\mathbf{u}}^{n+1}+j \mathbf{u}^{n} \cdot \nabla \mathbf{w}^{n} \cdot \mathbf{w}^{n+1}\right. \\
& \left.-2 v_{r} \tilde{\mathbf{u}}^{n+1} \nabla \times \mathbf{w}^{n}-2 v_{r} \mathbf{w}^{n+1} \nabla \times \tilde{\mathbf{u}}^{n}\right) d \mathbf{x} .
\end{aligned}
$$

Summing up (3.7), (3.8) and (3.9), we obtain

$$
\begin{aligned}
& \frac{1}{4 \Delta t}\left(\left\|\mathbf{u}^{n+1}\right\|^{2}-\left\|\mathbf{u}^{n}\right\|^{2}+\left\|\tilde{\mathbf{u}}^{n+1}-\mathbf{u}^{n}\right\|^{2}\right) \\
& \quad+\frac{j}{4 \Delta t}\left(\left\|\mathbf{w}^{n+1}\right\|^{2}-\left\|\mathbf{w}^{n}\right\|^{2}+\left\|\mathbf{w}^{n+1}-\mathbf{w}^{n}\right\|^{2}\right) \\
& \quad+\frac{1}{\Delta t}\left(\left|R^{n+1}\right|^{2}-\left|R^{n}\right|^{2}+\left|R^{n+1}-R^{n}\right|^{2}\right)+\frac{\Delta t}{2}\left\|\nabla p^{n+1}\right\|^{2}-\frac{\Delta t}{2}\left\|\nabla p^{n}\right\|^{2} \\
& =-\left(v_{0}\left\|\nabla \tilde{\mathbf{u}}^{n+1}\right\|^{2}+c_{1}\left\|\nabla \mathbf{w}^{n+1}\right\|^{2}+c_{2}\left\|\nabla \cdot \mathbf{w}^{n+1}\right\|^{2}+4 v_{r}\left\|\mathbf{w}^{n+1}\right\|^{2}\right),
\end{aligned}
$$

which implies the desired result (3.1).

Remark 3.1. Note that $\mathcal{E}^{n+1}-C_{0}$ defined in (3.2) is an approximation to the total energy, and $\left|R^{n+1}\right|^{2}-C_{0}$ is an approximation to half of the total energy. In particular, $\mathcal{E}^{n+1}$ includes the norms $\frac{1}{4}\left\|\mathbf{u}^{n+1}\right\|^{2}+\frac{j}{4}\left\|\mathbf{w}^{n+1}\right\|^{2}$ which will be essential in the convergence and error analysis of the scheme.

Next, we consider the second-order scheme for which the proof of energy stability is much more delicate.

Theorem 3.2. In the absence of the external force $f$ and $g$, the scheme (2.14) satisfies the following property unconditionally:

$$
\mathcal{E}^{n+1}-\mathcal{E}^{n} \leq-\Delta t\left(v_{0}\left(1-\frac{\chi}{2}\right)\left\|\nabla \tilde{\boldsymbol{u}}^{n+1}\right\|^{2}+c_{1}\left\|\nabla \boldsymbol{w}^{n+1}\right\|^{2}+c_{2}\left\|\nabla \cdot \boldsymbol{w}^{n+1}\right\|^{2}+4 v_{r}\left\|\boldsymbol{w}^{n+1}\right\|^{2}\right),
$$


where

$$
\begin{aligned}
\mathcal{E}^{n+1}= & \frac{1}{8}\left(\left\|\boldsymbol{u}^{n+1}\right\|^{2}+\left\|2 \boldsymbol{u}^{n+1}-\boldsymbol{u}^{n}\right\|^{2}\right)+\frac{j}{8}\left(\left\|\boldsymbol{w}^{n+1}\right\|^{2}+\left\|2 \boldsymbol{w}^{n+1}-\boldsymbol{w}^{n}\right\|^{2}\right) \\
& +\frac{1}{2}\left(\left|R^{n+1}\right|^{2}+\left|2 R^{n+1}-R^{n}\right|^{2}\right)+\frac{\Delta t^{2}}{3}\left\|\nabla\left(p^{n+1}+q^{n+1}\right)\right\|^{2}+\frac{\Delta t}{2 \chi v_{0}}\left\|q^{n+1}\right\|^{2} .
\end{aligned}
$$

Proof. Using the identity

$$
2(3 a-4 b+c, a)=|a|^{2}+|2 a-b|^{2}-|b|^{2}-|2 b-c|^{2}+|a-2 b+c|^{2},
$$

we can obtain

$$
\begin{aligned}
& \left(\frac{3 \tilde{\mathbf{u}}^{n+1}-4 \mathbf{u}^{n}+\mathbf{u}^{n-1}}{2 \Delta t}, \tilde{\mathbf{u}}^{n+1}\right) \\
= & \frac{3}{2 \Delta t}\left(\tilde{\mathbf{u}}^{n+1}-\mathbf{u}^{n+1}, \tilde{\mathbf{u}}^{n+1}\right)+\frac{1}{2 \Delta t}\left(3 \mathbf{u}^{n+1}-4 \mathbf{u}^{n}+\mathbf{u}^{n-1}, \tilde{\mathbf{u}}^{n+1}-\mathbf{u}^{n+1}\right) \\
& +\frac{1}{2 \Delta t}\left(3 \mathbf{u}^{n+1}-4 \mathbf{u}^{n}+\mathbf{u}^{n-1}, \mathbf{u}^{n+1}\right) \\
= & \frac{3}{4 \Delta t}\left(\left\|\tilde{\mathbf{u}}^{n+1}\right\|^{2}-\left\|\mathbf{u}^{n+1}\right\|^{2}+\left\|\tilde{\mathbf{u}}^{n+1}-\mathbf{u}^{n+1}\right\|^{2}\right) \\
& +\frac{1}{2 \Delta t}\left(3 \mathbf{u}^{n+1}-4 \mathbf{u}^{n}+\mathbf{u}^{n-1}, \mathbf{u}^{n+1}\right) \\
= & \frac{3}{4 \Delta t}\left(\left\|\tilde{\mathbf{u}}^{n+1}\right\|^{2}-\left\|\mathbf{u}^{n+1}\right\|^{2}+\left\|\tilde{\mathbf{u}}^{n+1}-\mathbf{u}^{n+1}\right\|^{2}\right) \\
& +\frac{1}{4 \Delta t}\left(\left\|\mathbf{u}^{n+1}\right\|^{2}+\left\|2 \mathbf{u}^{n+1}-\mathbf{u}^{n}\right\|^{2}-\left\|\mathbf{u}^{n}\right\|^{2}-\left\|2 \mathbf{u}^{n}-\mathbf{u}^{n-1}\right\|^{2}\right. \\
& \left.+\left\|\mathbf{u}^{n+1}-2 \mathbf{u}^{n}+\mathbf{u}^{n-1}\right\|^{2}\right) .
\end{aligned}
$$

Then, taking the inner product of (2.14a) with $\tilde{\mathbf{u}}^{n+1}$ and using the above, we obtain

$$
\begin{aligned}
& \frac{3}{4 \Delta t}\left(\left\|\tilde{\mathbf{u}}^{n+1}\right\|^{2}-\left\|\mathbf{u}^{n+1}\right\|^{2}+\left\|\tilde{\mathbf{u}}^{n+1}-\mathbf{u}^{n+1}\right\|^{2}\right) \\
& \quad+\frac{1}{4 \Delta t}\left(\left\|\mathbf{u}^{n+1}\right\|^{2}+\left\|2 \mathbf{u}^{n+1}-\mathbf{u}^{n}\right\|^{2}-\left\|\mathbf{u}^{n}\right\|^{2}-\left\|2 \mathbf{u}^{n}-\mathbf{u}^{n-1}\right\|^{2}\right. \\
& \left.\quad+\left\|\mathbf{u}^{n+1}-2 \mathbf{u}^{n}+\mathbf{u}^{n-1}\right\|^{2}\right) \\
& \quad+\frac{R^{n+1}}{\sqrt{\bar{E}^{n+1}}}\left(\overline{\mathbf{u}}^{n+1} \cdot \nabla \overline{\mathbf{u}}^{n+1}, \tilde{\mathbf{u}}^{n+1}\right)+v_{0}\left\|\nabla \tilde{\mathbf{u}}^{n+1}\right\|^{2}+\left(\nabla p^{n}, \tilde{\mathbf{u}}^{n+1}\right) \\
& =2 v_{r} \frac{R^{n+1}}{\sqrt{\bar{E}^{n+1}}}\left(\nabla \times \overline{\mathbf{w}}^{n+1}, \tilde{\mathbf{u}}^{n+1}\right) .
\end{aligned}
$$

Similarly as in [19], we introduce a sequence $\left\{q^{n}\right\}$ defined by

$$
q^{n}=\chi v_{0} \nabla \cdot \tilde{\mathbf{u}}^{n}+q^{n-1} \quad \text { with } \quad q^{-1}=q^{0}=0,
$$


and rewrite $(2.14 \mathrm{~b})$ as

$$
\sqrt{3} \mathbf{u}^{n+1}+\frac{2}{\sqrt{3}} \Delta t \nabla\left(p^{n+1}+q^{n+1}\right)=\sqrt{3} \tilde{\mathbf{u}}^{n+1}+\frac{2}{\sqrt{3}} \Delta t \nabla\left(p^{n}+q^{n}\right) .
$$

Taking the inner product of above with itself on both sides, we get

$$
\begin{aligned}
& 3\left\|\mathbf{u}^{n+1}\right\|^{2}+\frac{4}{3} \Delta t^{2}\left\|\nabla\left(p^{n+1}+q^{n+1}\right)\right\|^{2} \\
= & 3\left\|\tilde{\mathbf{u}}^{n+1}\right\|^{2}+4 \Delta t\left(\tilde{\mathbf{u}}^{n+1}, \nabla\left(p^{n}+q^{n}\right)\right)+\frac{4}{3} \Delta t^{2}\left\|\nabla\left(p^{n}+q^{n}\right)\right\|^{2} .
\end{aligned}
$$

Notice that

$$
\begin{aligned}
4 \Delta t\left(\tilde{\mathbf{u}}^{n+1}, \nabla\left(p^{n}+q^{n}\right)\right) & =4 \Delta t\left(\tilde{\mathbf{u}}^{n+1}, \nabla p^{n}\right)-\frac{4 \Delta t}{\chi v_{0}}\left(q^{n+1}-q^{n}, q^{n}\right) \\
& =4 \Delta t\left(\tilde{\mathbf{u}}^{n+1}, \nabla p^{n}\right)+\frac{2 \Delta t}{\chi v_{0}}\left(\left\|q^{n}\right\|^{2}-\left\|q^{n+1}\right\|^{2}+\left\|q^{n+1}-q^{n}\right\|^{2}\right) .
\end{aligned}
$$

Thanks to (3.15), we have

$$
\frac{2 \Delta t}{\chi v_{0}}\left\|q^{n+1}-q^{n}\right\|^{2}=2 \chi v_{0} \Delta t\left\|\nabla \cdot \tilde{\mathbf{u}}^{n+1}\right\|^{2} \leq 2 \chi v_{0} \Delta t\left\|\nabla \tilde{\mathbf{u}}^{n+1}\right\|^{2}
$$

Combining (3.16) with (3.17) and (3.18), we get

$$
\begin{aligned}
& \frac{3}{4 \Delta t}\left\|\mathbf{u}^{n+1}\right\|^{2}-\frac{3}{4 \Delta t}\left\|\tilde{\mathbf{u}}^{n+1}\right\|^{2}+\frac{\Delta t}{3}\left\|\nabla\left(p^{n+1}+q^{n+1}\right)\right\|^{2}-\frac{\Delta t}{3}\left\|\nabla\left(p^{n}+q^{n}\right)\right\|^{2} \\
= & \left(\tilde{\mathbf{u}}^{n+1}, \nabla p^{n}\right)+\frac{1}{2 \chi v_{0}}\left(\left\|q^{n}\right\|^{2}-\left\|q^{n+1}\right\|^{2}\right)+\frac{\chi v_{0}}{2}\left\|\nabla \cdot \tilde{\mathbf{u}}^{n+1}\right\|^{2} .
\end{aligned}
$$

We then derive from (3.14) and (3.19) that

$$
\begin{aligned}
& \frac{1}{4 \Delta t}\left(\left\|\mathbf{u}^{n+1}\right\|^{2}+\left\|2 \mathbf{u}^{n+1}-\mathbf{u}^{n}\right\|^{2}-\left\|\mathbf{u}^{n}\right\|^{2}-\left\|2 \mathbf{u}^{n}-\mathbf{u}^{n-1}\right\|^{2}\right. \\
& \left.+\left\|\mathbf{u}^{n+1}-2 \mathbf{u}^{n}+\mathbf{u}^{n-1}\right\|^{2}+3\left\|\tilde{\mathbf{u}}^{n+1}-\mathbf{u}^{n+1}\right\|^{2}\right) \\
& +\frac{\Delta t}{3}\left\|\nabla\left(p^{n+1}+q^{n+1}\right)\right\|^{2}-\frac{\Delta t}{3}\left\|\nabla\left(p^{n}+q^{n}\right)\right\|^{2}+\frac{1}{2 \chi v_{0}}\left\|q^{n+1}\right\|^{2}-\frac{1}{2 \chi v_{0}}\left\|q^{n}\right\|^{2} \\
& \quad+\frac{R^{n+1}}{\sqrt{\bar{E}^{n+1}}}\left(\overline{\mathbf{u}}^{n+1} \cdot \nabla \overline{\mathbf{u}}^{n+1}, \tilde{\mathbf{u}}^{n+1}\right)+v_{0}\left\|\nabla \tilde{\mathbf{u}}^{n+1}\right\|^{2} \\
& \leq \frac{\chi v_{0}}{2}\left\|\nabla \tilde{\mathbf{u}}^{n+1}\right\|^{2}+2 v_{r} \frac{R^{n+1}}{\sqrt{\bar{E}^{n+1}}}\left(\nabla \times \overline{\mathbf{w}}^{n+1}, \tilde{\mathbf{u}}^{n+1}\right) .
\end{aligned}
$$


Next, we take the inner product of (2.14c) with $\mathbf{w}^{n+1}$, and use (3.13) to obtain

$$
\begin{aligned}
& \frac{j}{4 \Delta t}\left(\left\|\mathbf{w}^{n+1}\right\|^{2}+\left\|2 \mathbf{w}^{n+1}-\mathbf{w}^{n}\right\|^{2}-\left\|\mathbf{w}^{n}\right\|^{2}-\left\|2 \mathbf{w}^{n}-\mathbf{w}^{n-1}\right\|^{2}+\left\|\mathbf{w}^{n+1}-2 \mathbf{w}^{n}+\mathbf{w}^{n-1}\right\|^{2}\right) \\
& +j \frac{R^{n+1}}{\sqrt{\bar{E}^{n+1}}}\left(\overline{\mathbf{u}}^{n+1} \cdot \nabla \overline{\mathbf{w}}^{n+1}, \mathbf{w}^{n+1}\right)+c_{1}\left\|\nabla \mathbf{w}^{n+1}\right\|^{2}+c_{2}\left\|\nabla \cdot \mathbf{w}^{n+1}\right\|^{2}+4 v_{r}\left\|\mathbf{w}^{n+1}\right\|^{2} \\
= & 2 v_{r} \frac{R^{n+1}}{\sqrt{\bar{E}^{n+1}}}\left(\nabla \times \overline{\tilde{\mathbf{u}}}^{n+1}, \mathbf{w}^{n+1}\right) .
\end{aligned}
$$

Using again (3.13), we derive from (2.14d) that

$$
\begin{aligned}
& \frac{1}{2 \Delta t}\left(\left|R^{n+1}\right|^{2}+\left|2 R^{n+1}-R^{n}\right|^{2}-\left|R^{n}\right|^{2}-\left|2 R^{n}-R^{n-1}\right|^{2}+\left|R^{n+1}-2 R^{n}+R^{n-1}\right|^{2}\right) \\
= & \frac{1}{8 \Delta t}\left(\left\|\mathbf{u}^{n+1}\right\|^{2}+\left\|2 \mathbf{u}^{n+1}-\mathbf{u}^{n}\right\|^{2}-\left\|\mathbf{u}^{n}\right\|^{2}-\left\|2 \mathbf{u}^{n}-\mathbf{u}^{n-1}\right\|^{2}+\left\|\mathbf{u}^{n+1}-2 \mathbf{u}^{n}+\mathbf{u}^{n-1}\right\|^{2}\right) \\
& +\frac{j}{8 \Delta t}\left(\left\|\mathbf{w}^{n+1}\right\|^{2}+\left\|2 \mathbf{w}^{n+1}-\mathbf{w}^{n}\right\|^{2}-\left\|\mathbf{w}^{n}\right\|^{2}-\left\|2 \mathbf{w}^{n}-\mathbf{w}^{n-1}\right\|^{2}+\left\|\mathbf{w}^{n+1}-2 \mathbf{w}^{n}+\mathbf{w}^{n-1}\right\|^{2}\right) \\
& +\int_{\Omega} \frac{R^{n+1}}{\sqrt{\overline{\bar{E}^{n+1}}}}\left(\overline{\mathbf{u}}^{n+1} \cdot \nabla \overline{\mathbf{u}}^{n+1} \cdot \tilde{\mathbf{u}}^{n+1}+j \overline{\mathbf{u}}^{n+1} \cdot \nabla \overline{\mathbf{w}}^{n+1} \cdot \mathbf{w}^{n+1}\right. \\
& \left.-2 v_{r} \tilde{\mathbf{u}}^{n+1} \nabla \times \overline{\mathbf{w}}^{n+1}-2 v_{r} \mathbf{w}^{n+1} \nabla \times \overline{\mathbf{u}}^{n+1}\right) d \mathbf{x} .
\end{aligned}
$$

Summing up the results of (3.20), (3.21) and (3.22), we arrive at

$$
\begin{aligned}
& \frac{1}{8 \Delta t}\left(\left\|\mathbf{u}^{n+1}\right\|^{2}+\left\|2 \mathbf{u}^{n+1}-\mathbf{u}^{n}\right\|^{2}-\left\|\mathbf{u}^{n}\right\|^{2}-\left\|2 \mathbf{u}^{n}-\mathbf{u}^{n-1}\right\|^{2}\right) \\
& \quad+\frac{j}{8 \Delta t}\left(\left\|\mathbf{w}^{n+1}\right\|^{2}+\left\|2 \mathbf{w}^{n+1}-\mathbf{w}^{n}\right\|^{2}-\left\|\mathbf{w}^{n}\right\|^{2}-\left\|2 \mathbf{w}^{n}-\mathbf{w}^{n-1}\right\|^{2}\right) \\
& \quad+\frac{1}{2 \Delta t}\left(\left|R^{n+1}\right|^{2}+\left|2 R^{n+1}-R^{n}\right|^{2}-\left|R^{n}\right|^{2}-\left|2 R^{n}-R^{n-1}\right|^{2}\right) \\
& \quad+\frac{\Delta t}{3}\left\|\nabla\left(p^{n+1}+q^{n+1}\right)\right\|^{2}-\frac{\Delta t}{3}\left\|\nabla\left(p^{n}+q^{n}\right)\right\|^{2}+\frac{1}{2 \chi v_{0}}\left\|q^{n+1}\right\|^{2}-\frac{1}{2 \chi v_{0}}\left\|q^{n}\right\|^{2} \\
& \leq-\left(v_{0}\left(1-\frac{\chi}{2}\right)\left\|\nabla \tilde{\mathbf{u}}^{n+1}\right\|^{2}+c_{1}\left\|\nabla \mathbf{w}^{n+1}\right\|^{2}+c_{2}\left\|\nabla \cdot \mathbf{w}^{n+1}\right\|^{2}+4 v_{r}\left\|\mathbf{w}^{n+1}\right\|^{2}\right),
\end{aligned}
$$

which implies the desired result (3.12) since $0<\chi \leq 1$.

\section{Fully discrete schemes with a spectral discretization in space}

Unlike the SAV schemes for gradient flows [24], the stability results in the last section does not automatically carry over to all Galerkin spatial discretizations. Note that in the proofs of Theorems 3.1 and 3.2, the equations for $\tilde{\mathbf{u}}^{n+1}$ and $\mathbf{w}^{n+1}$ in the schemes (2.9) and 
(2.14) are interpreted as weakly satisfied, while the equation for $\left(\mathbf{u}^{n+1}, p^{n+1}\right)$ are interpreted as strongly (pointwise) satisfied. Hence, the main difficulty is to derive the discrete analogs of (3.6) and (3.16) which are obtained from the fact that (2.9b) and (2.14b) are satisfied everywhere in the spatially continuous case.

We observe that using the usual $P_{N} \times P_{N-2}$ spectral discretization for Stokes problem [2] can not satisfy (3.6) and (3.16) pointwise so the proofs of Theorems 3.1 and 3.2 can not carry over to the discrete case. Indeed, $(2.9 \mathrm{~b})$ corresponds to a different mixed problem (cf. for instance, $[1,3]$ ) than the usual Stokes problem. For the sake of efficiency, we seek a space discretization of its equivalent formulation (in the space continuous case) (2.12b). From the proof of Theorem 3.1, we see that the key to preserve the stability in the fully discrete case is that (3.5) and (3.6) are also satisfied in the fully discrete case. Note that with (3.5), we can derive (3.6) if

$$
\left(\nabla p^{k+1}, \mathbf{u}^{k+1}\right)=0 .
$$

Therefore, we need to choose fully discretization of (2.12b) such that the discrete versions of (3.5) and (4.1) are satisfied. To this end, we consider a spectral discretization for the space variables as follows. Let $\Omega=(-1,1)^{d}$, and $P_{N}$ be the space of polynomials of degree less than or equal to $N$ in each direction. We denote

$$
\mathbf{X}_{N}=\left(P_{N}\right)^{d}, \quad X_{N}^{0}=\operatorname{span}\left\{\mathbf{v} \in P_{N}:\left.v\right|_{\partial \Omega}=0\right\}, \quad \mathbf{X}_{N}^{0}=\left(X_{N}^{0}\right)^{d},
$$

and discretize (2.12b) as follows: given $p_{N}^{n} \in P_{N}$ and $\tilde{\mathbf{u}}_{N}^{n+1} \in X_{N}^{0}$, find $p_{N}^{n+1} \in P_{N}$ such that

$$
\left(\nabla\left(p_{N}^{n+1}-p_{N}^{n}\right), \nabla m_{N}\right)=\frac{1}{\Delta t}\left(\tilde{\mathbf{u}}_{N}^{n+1}, \nabla m_{N}\right), \quad \forall m_{N} \in P_{N},
$$

which is a discrete Poisson equation in the weak form for $p_{N}^{n+1}-p_{N}^{n}$, and set

$$
\mathbf{u}_{N}^{n+1}=\tilde{\mathbf{u}}_{N}^{n+1}-\Delta t \nabla\left(p_{N}^{n+1}-p_{N}^{n}\right) .
$$

Obviously $\mathbf{u}_{N}^{n+1} \in X_{N}$. We derive from (4.3)-(4.4) that

$$
\left(\mathbf{u}_{N}^{n+1}, \nabla m_{N}\right)=0, \quad \forall m_{N} \in P_{N} .
$$

We then derive from the above and (4.4) that

$$
\left\|\mathbf{u}_{N}^{n+1}\right\|^{2}+\Delta t^{2}\left\|\nabla p_{N}^{n+1}\right\|^{2}=\left\|\tilde{\mathbf{u}}_{N}^{n+1}\right\|^{2}+2 \Delta t\left(\tilde{\mathbf{u}}_{N}^{n+1}, \nabla p_{N}^{n}\right)+\Delta t^{2}\left\|\nabla p_{N}^{n}\right\|^{2},
$$

which is the discrete analog of (3.6).

We can now construct a fully discrete version of the scheme (2.9).

Find $\tilde{\mathbf{u}}_{N}^{n+1} \in \mathbf{X}_{N}^{0},\left(\mathbf{u}_{N}^{n+1}, p_{N}^{n+1}\right) \in\left(\mathbf{X}_{N}, P_{N}\right), \mathbf{w}_{N}^{n+1} \in \mathbf{X}_{N}^{0}$ and $R_{N}^{n+1} \in \mathbb{R}$ such that

$$
\begin{aligned}
& \frac{1}{\Delta t}\left(\tilde{\mathbf{u}}_{N}^{n+1}-\mathbf{u}_{N}^{n}, \mathbf{v}_{N}\right)+v_{0}\left(\nabla \tilde{\mathbf{u}}_{N}^{n+1}, \nabla \mathbf{v}_{N}\right) \\
= & \frac{R_{N}^{n+1}}{\sqrt{E_{N}^{n}}}\left(2 v_{r} \nabla \times \mathbf{w}_{N}^{n}-\mathbf{u}_{N}^{n} \cdot \nabla \mathbf{u}_{N}^{n}, \mathbf{v}_{N}\right)-\left(\nabla p_{N}^{n}-\mathbf{f}^{n+1}, \mathbf{v}_{N}\right), \quad \forall \mathbf{v}_{N} \in \mathbf{X}_{N}^{0} ;
\end{aligned}
$$




$$
\begin{aligned}
& \left(\nabla\left(p_{N}^{n+1}-p_{N}^{n}\right), \nabla m_{N}\right)=\frac{1}{\Delta t}\left(\tilde{\mathbf{u}}_{N}^{n+1}, \nabla m_{N}\right), \quad \forall m_{N} \in P_{N}, \\
& \quad \mathbf{u}_{N}^{n+1}=\tilde{\mathbf{u}}_{N}^{n+1}-\Delta t \nabla\left(p_{N}^{n+1}-p_{N}^{n}\right) ; \\
& \frac{j}{\Delta t}\left(\mathbf{w}_{N}^{n+1}-\mathbf{w}_{N}^{n}, \mathbf{v}_{N}\right)+c_{1}\left(\nabla \mathbf{w}_{N}^{n+1}, \nabla \mathbf{v}_{N}\right)+c_{2}\left(\nabla \cdot \mathbf{w}_{N}^{n+1}, \nabla \cdot \mathbf{v}_{N}\right)+4 v_{r}\left(\mathbf{w}_{N}^{n+1}, \mathbf{v}_{N}\right) \\
& =\frac{R_{N}^{n+1}}{\sqrt{E_{N}^{n}}}\left(2 v_{r} \nabla \times \tilde{\mathbf{u}}_{N}^{n}-j \mathbf{u}_{N}^{n} \cdot \nabla \mathbf{w}_{N}^{n}, \mathbf{v}_{N}\right)+\left(\mathbf{g}^{n+1}, \mathbf{v}_{N}\right), \quad \forall \mathbf{v}_{N} \in \boldsymbol{X}_{N}^{0} ; \\
& 2 R_{N}^{n+1} \frac{R_{N}^{n+1}-R_{N}^{n}}{\Delta t}=\int_{\Omega} \frac{1}{2}\left(\mathbf{u}_{N}^{n+1} \frac{\mathbf{u}_{N}^{n+1}-\mathbf{u}_{N}^{n}}{\Delta t}+j \mathbf{w}_{N}^{n+1} \frac{\mathbf{w}_{N}^{n+1}-\mathbf{w}_{N}^{n}}{\Delta t}\right) \\
& \quad+\frac{R_{N}^{n+1}}{\sqrt{E_{N}^{n}}}\left(\mathbf{u}_{N}^{n} \cdot \nabla \mathbf{u}_{N}^{n} \cdot \tilde{\mathbf{u}}_{N}^{n+1}+j \mathbf{u}_{N}^{n} \cdot \nabla \mathbf{w}_{N}^{n} \cdot \mathbf{w}_{N}^{n+1}\right. \\
& \left.\quad-2 v_{r} \tilde{\mathbf{u}}_{N}^{n+1} \nabla \times \mathbf{w}_{N}^{n}-2 v_{r} \mathbf{w}_{N}^{n+1} \nabla \times \tilde{\mathbf{u}}_{N}^{n}\right) d \mathbf{x} ;
\end{aligned}
$$

where

$$
E_{N}^{n}=\frac{1}{4}\left(\left\|\mathbf{u}_{N}^{n}\right\|^{2}+j\left\|\mathbf{w}_{N}^{n}\right\|^{2}\right)+C_{0}
$$

The implementation procedure of the above scheme is exactly the same as in the spatial continuous case. More precisely, at each time step, one only has to solve a sequence of Poisson-type equations for $\tilde{\mathbf{u}}_{N}^{n+1}$ and $p_{N}^{n+1}$, and a second-order equations with constantcoefficients for $\mathbf{w}_{N}^{n+1}$. Note that (4.6b) is indeed a discrete Poisson equation in weak form. These Poisson type equations and second-order equations with constant-coefficients can be solved efficiently by using the matrix-diagonalization technique $[4,23]$.

Therefore, by following exactly the same procedure as in the proof of Theorem 3.1, and thanks to (4.5), we can prove the following:

Corollary 4.1. In the absence of the external force $f$ and $g$, the scheme (4.6) satisfies the following discrete energy law unconditionally:

$$
\mathcal{E}_{N}^{n+1}-\mathcal{E}_{N}^{n} \leq-\Delta t\left(v_{0}\left\|\nabla \tilde{\boldsymbol{u}}_{N}^{n+1}\right\|^{2}+c_{1}\left\|\nabla \boldsymbol{w}_{N}^{n+1}\right\|^{2}+c_{2}\left\|\nabla \cdot \boldsymbol{w}_{N}^{n+1}\right\|^{2}+4 v_{r}\left\|\boldsymbol{w}_{N}^{n+1}\right\|^{2}\right),
$$

where

$$
\mathcal{E}_{N}^{n+1}=\frac{1}{4}\left\|\boldsymbol{u}_{N}^{n+1}\right\|^{2}+\frac{j}{4}\left\|\boldsymbol{w}_{N}^{n+1}\right\|^{2}+\left|R_{N}^{n+1}\right|^{2}+\frac{\Delta t^{2}}{2}\left\|\nabla p_{N}^{n+1}\right\|^{2} .
$$

Similarly, a fully discrete version of the second-order scheme (2.14) is as follows. Find $\tilde{\mathbf{u}}_{N}^{n+1} \in \mathbf{X}_{N}^{0},\left(\mathbf{u}_{N}^{n+1}, p_{N}^{n+1}\right) \in\left(\mathbf{X}_{N}, P_{N}\right), \mathbf{w}_{N}^{n+1} \in \mathbf{X}_{N}^{0}$ and $R_{N}^{n+1} \in \mathbb{R}$ such that

$$
\begin{aligned}
& \frac{1}{2 \Delta t}\left(3 \tilde{\mathbf{u}}_{N}^{n+1}-4 \mathbf{u}_{N}^{n}+\mathbf{u}_{N}^{n-1}, \mathbf{v}_{N}\right)+v_{0}\left(\nabla \tilde{\mathbf{u}}_{N}^{n+1}, \nabla \mathbf{v}_{N}\right) \\
= & \frac{R_{N}^{n+1}}{\sqrt{\bar{E}_{N}^{n+1}}}\left(2 v_{r} \nabla \times \overline{\mathbf{w}}_{N}^{n+1}-\overline{\mathbf{u}}_{N}^{n+1} \cdot \nabla \overline{\mathbf{u}}_{N}^{n+1}, \mathbf{v}_{N}\right)-\left(\nabla p_{N}^{n}-\mathbf{f}^{n+1}, \mathbf{v}_{N}\right), \quad \forall \mathbf{v}_{N} \in \mathbf{X}_{N}^{0}
\end{aligned}
$$




$$
\begin{aligned}
& \left(\nabla\left(p_{N}^{n+1}-p_{N}^{n}+\chi v_{0} \nabla \tilde{\mathbf{u}}_{N}^{n+1}\right), \nabla m_{N}\right)=\frac{3}{2 \Delta t}\left(\tilde{\mathbf{u}}_{N}^{n+1}, \nabla m_{N}\right), \quad \forall m_{N} \in P_{N}, \\
& \quad \mathbf{u}_{N}^{n+1}=\tilde{\mathbf{u}}_{N}^{n+1}-\frac{2 \Delta t}{3} \nabla\left(p_{N}^{n+1}-p_{N}^{n}+\chi v_{0} \nabla \tilde{\mathbf{u}}_{N}^{n+1}\right) ; \\
& \frac{j}{2 \Delta t}\left(3 \mathbf{w}_{N}^{n+1}-4 \mathbf{w}_{N}^{n}+\mathbf{w}_{N}^{n-1}, \mathbf{v}_{N}\right)+c_{1}\left(\nabla \mathbf{w}_{N}^{n+1}, \nabla \mathbf{v}_{N}\right)+c_{2}\left(\nabla \cdot \mathbf{w}_{N}^{n+1}, \nabla \cdot \mathbf{v}_{N}\right)+4 v_{r}\left(\mathbf{w}_{N}^{n+1}, \mathbf{v}_{N}\right) \\
& =\frac{R^{n+1}}{\sqrt{\bar{E}^{n+1}}}\left(2 v_{r} \nabla \times \overline{\mathbf{u}}_{N}^{n+1}-j \overline{\mathbf{u}}_{N}^{n+1} \cdot \nabla \overline{\mathbf{w}}_{N}^{n+1}, \mathbf{v}_{N}\right)+\left(\mathbf{g}^{n+1}, \mathbf{v}_{N}\right), \quad \forall \mathbf{v}_{N} \in \mathbf{X}_{N}^{0} \\
& 2 R_{N}^{n+1} \frac{3 R_{N}^{n+1}-4 R_{N}^{n}+R_{N}^{n-1}}{2 \Delta t} \\
& =\int_{\Omega} \frac{1}{2}\left(\mathbf{u}_{N}^{n+1} \frac{3 \mathbf{u}_{N}^{n+1}-4 \mathbf{u}_{N}^{n}+\mathbf{u}_{N}^{n-1}}{2 \Delta t}+j \mathbf{w}_{N}^{n+1} \frac{3 \mathbf{w}_{N}^{n+1}-4 \mathbf{w}_{N}^{n}+\mathbf{w}_{N}^{n-1}}{2 \Delta t}\right) \\
& \quad+\frac{R_{N}^{n+1}}{\sqrt{\bar{E}_{N}^{n+1}}}\left(\overline{\mathbf{u}}_{N}^{n+1} \cdot \nabla \overline{\mathbf{u}}_{N}^{n+1} \cdot \tilde{\mathbf{u}}_{N}^{n+1}+j \overline{\mathbf{u}}_{N}^{n+1} \cdot \nabla \overline{\mathbf{w}}_{N}^{n+1} \cdot \mathbf{w}_{N}^{n+1}\right. \\
& \left.\quad-2 v_{r} \tilde{\mathbf{u}}_{N}^{n+1} \nabla \times \overline{\mathbf{w}}_{N}^{n+1}-2 v_{r} \mathbf{w}_{N}^{n+1} \nabla \times \overline{\mathbf{u}}_{N}^{n+1}\right) d \mathbf{x}
\end{aligned}
$$

where $\bar{E}_{N}^{n+1}=2 E_{N}^{n}-E_{N}^{n-1}$ with $E_{N}^{k}$ defined in (4.7).

We observe that $(4.10 \mathrm{~b})$ is still a discrete Poisson equation in weak form, so the above scheme can be efficiently implemented exactly as in the first-order case.

In order to prove the stability, we observe from $(2.14 b)$ that

$$
\left(\mathbf{u}_{N}^{n+1}, \nabla m_{N}\right)=0, \quad \forall m_{N} \in P_{N} .
$$

On the other hand, setting

$$
q_{N}^{n}=\chi v_{0} \nabla \cdot \tilde{\mathbf{u}}_{N}^{n}+q_{N}^{n-1} \quad \text { with } \quad q_{N}^{-1}=q_{N}^{0}=0,
$$

we can rewrite the second equation in $(2.14 \mathrm{~b})$ as

$$
\mathbf{u}_{N}^{n+1}+\frac{2}{3} \Delta t \nabla\left(p_{N}^{n+1}+q_{N}^{n+1}\right)=\tilde{\mathbf{u}}_{N}^{n+1}+\frac{2}{3} \Delta t \nabla\left(p_{N}^{n}+q_{N}^{n}\right)
$$

We then derive from (4.11)-(4.13) that

$$
\begin{aligned}
& 3\left\|\mathbf{u}_{N}^{n+1}\right\|^{2}+\frac{4}{3} \Delta t^{2}\left\|\nabla\left(p_{N}^{n+1}+q_{N}^{n+1}\right)\right\|^{2} \\
= & 3\left\|\tilde{\mathbf{u}}_{N}^{n+1}\right\|^{2}+4 \Delta t\left(\tilde{\mathbf{u}}_{N}^{n+1}, \nabla\left(p_{N}^{n}+q_{N}^{n}\right)\right)+\frac{4}{3} \Delta t^{2}\left\|\nabla\left(p_{N}^{n}+q_{N}^{n}\right)\right\|^{2},
\end{aligned}
$$

which is the discrete analog of (3.16).

Therefore, by following exactly the same procedure as in the proof of Theorem 3.2, and thanks to (4.14), we can prove the following: 
Corollary 4.2. In the absence of the external force $f$ and $g$, the scheme (4.10) satisfies the following property unconditionally:

$$
\mathcal{E}_{N}^{n+1}-\mathcal{E}_{N}^{n} \leq-\Delta t\left(v_{0}\left(1-\frac{\chi}{2}\right)\left\|\nabla \tilde{\boldsymbol{u}}_{N}^{n+1}\right\|^{2}+c_{1}\left\|\nabla \boldsymbol{w}_{N}^{n+1}\right\|^{2}+c_{2}\left\|\nabla \cdot \boldsymbol{w}_{N}^{n+1}\right\|^{2}+4 v_{r}\left\|\boldsymbol{w}_{N}^{n+1}\right\|^{2}\right),
$$

where

$$
\begin{aligned}
\mathcal{E}_{N}^{n+1}= & \frac{1}{8}\left(\left\|\boldsymbol{u}_{N}^{n+1}\right\|^{2}+\left\|2 \boldsymbol{u}_{N}^{n+1}-\boldsymbol{u}_{N}^{n}\right\|^{2}\right)+\frac{j}{8}\left(\left\|\boldsymbol{w}_{N}^{n+1}\right\|^{2}+\left\|2 \boldsymbol{w}_{N}^{n+1}-\boldsymbol{w}_{N}^{n}\right\|^{2}\right) \\
& +\frac{1}{2}\left(\left|R_{N}^{n+1}\right|^{2}+\left|2 R_{N}^{n+1}-R_{N}^{n}\right|^{2}\right)+\frac{\Delta t^{2}}{3}\left\|\nabla\left(p_{N}^{n+1}+q_{N}^{n+1}\right)\right\|^{2}+\frac{\Delta t}{2 \chi v_{0}}\left\|q_{N}^{n+1}\right\|^{2} .
\end{aligned}
$$

\section{Numerical results}

In this section, we present some numerical results using the SAV schemes for the MNSE. We consider 2D micropolar fluids by assuming that the $x_{3}$-direction of the velocity component $u_{3}$ is zero, and the axes of rotation of particles are parallel to the $x_{3}$-axis $[5,14]$. Then the unknowns are

$$
\mathbf{u}(x, t)=\left(u_{1}(\mathbf{x}, t), u_{2}(\mathbf{x}, t)\right), \quad w=w(\mathbf{x}, t), \quad p=p(\mathbf{x}, t), \quad \mathbf{x}=\left(x_{1}, x_{2}\right),
$$

the external forcing functions are

$$
\mathbf{f}=\left(f_{1}(\mathbf{x}, t), f_{2}(\mathbf{x}, t)\right), \quad g=g(\mathbf{x}, t),
$$

and the system (1.1) becomes

$$
\left\{\begin{array}{l}
\mathbf{u}_{t}+\mathbf{u} \cdot \nabla \mathbf{u}-\left(v+v_{r}\right) \triangle \mathbf{u}+\nabla p=2 v_{r} \nabla \times w+\mathbf{f} \\
\nabla \cdot \mathbf{u}=0 \\
j w_{t}+j \mathbf{u} \cdot \nabla w-c_{1} \Delta w+4 v_{r} w=2 v_{r} \nabla \times \mathbf{u}+g
\end{array}\right.
$$

where

$$
\nabla \times \mathbf{u}=\frac{\partial u_{2}}{\partial x_{1}}-\frac{\partial u_{1}}{\partial x_{2}}, \quad \nabla \times w=\left(\frac{\partial w}{\partial x_{2}},-\frac{\partial w}{\partial x_{1}}\right)
$$

We consider a square domain $\Omega=(-1,1)^{2} \subset \mathbb{R}^{2}$ with Dirichlet boundary conditions on the linear velocity $\mathbf{u}$ and the angular velocity $\mathbf{w}$. We use the spectral spatial discretization described in the last section.

Unless otherwise specified, we take $C_{0}=0.01$. 


\subsection{Convergence tests}

We first verify the first- and second-order accuracy of the proposed numerical schemes. We fix the physical parameters to

$$
j=1, \quad c_{1}=2,
$$

and solve (1.1) with right hand sides $\mathbf{f}$ and $\mathbf{g}$ chosen so that the exact solution is

$$
\begin{aligned}
& \mathbf{u}(x, y, t)=\pi \sin (t)\left(\sin ^{2}(\pi x) \sin (2 \pi y),-\sin (2 \pi x) \sin ^{2}(\pi y)\right)^{T}, \\
& p(x, y, t)=\sin (t) \cos (\pi x) \sin (\pi y), \\
& \mathbf{w}(x, y, t)=\pi \sin (t) \sin ^{2}(\pi x) \sin ^{2}(\pi y) .
\end{aligned}
$$

We use $100 \times 100$ modes to discretize the space variables, so the spatial discretization error is negligible compared to the time discretization. We take $\chi=0.5$ and list the errors at $T=$ 0.2 in Tables 1-8. We observe that both Scheme I and Scheme II achieve the expected firstand second-order accuracy in time, respectively, except that the pressure convergence rate for the Scheme II is about 3/2 which is consistent with the error analysis in [9].

\subsection{Validation of energy dissipation}

In this example, we validate the unconditional energy dissipation of the proposed SAV scheme. We set the external body forces $\mathbf{f}=0, \mathbf{g}=0$.

Table 1: Error and convergence rates of Scheme I where $v=v_{r}=1$.

\begin{tabular}{||c|c|c|c|c|c|c||}
\hline$\delta t$ & $\|$ error $_{\mathbf{u}} \|_{\infty}$ & Rate & $\|$ error $_{p} \|_{2}$ & Rate & $\|$ error $_{\mathbf{w}} \|_{\infty}$ & Rate \\
\hline $1.25 \times 10^{-2}$ & $2.30 \mathrm{E}(-3)$ & - & $1.12 \mathrm{E}(-2)$ & - & $6.40 \mathrm{E}(-3)$ & - \\
$6.25 \times 10^{-3}$ & $1.10 \mathrm{E}(-3)$ & 1.01 & $6.00 \mathrm{E}(-3)$ & 0.91 & $3.20 \mathrm{E}(-3)$ & 0.99 \\
$3.125 \times 10^{-3}$ & $5.67 \mathrm{E}(-4)$ & 1.00 & $3.00 \mathrm{E}(-3)$ & 1.02 & $1.60 \mathrm{E}(-3)$ & 1.00 \\
$1.5625 \times 10^{-3}$ & $2.83 \mathrm{E}(-4)$ & 1.00 & $1.40 \mathrm{E}(-3)$ & 1.04 & $8.09 \mathrm{E}(-4)$ & 1.00 \\
$7.8125 \times 10^{-4}$ & $1.42 \mathrm{E}(-4)$ & 1.00 & $7.04 \mathrm{E}(-4)$ & 1.03 & $4.05 \mathrm{E}(-4)$ & 1.00 \\
\hline
\end{tabular}

Table 2: Error and convergence rates of Scheme II where $v=v_{r}=1$.

\begin{tabular}{||c|c|c|c|c|c|c||}
\hline$\delta t$ & $\|$ erro $_{\mathbf{u}} \|_{\infty}$ & Rate & $\|$ error $_{p} \|_{2}$ & Rate & $\|$ error $_{\mathbf{w}} \|_{\infty}$ & Rate \\
\hline $2.5 \times 10^{-2}$ & $3.81 \mathrm{E}(-4)$ & - & $1.86 \mathrm{E}(-3)$ & - & $7.22 \mathrm{E}(-5)$ & - \\
$1.25 \times 10^{-2}$ & $1.07 \mathrm{E}(-4)$ & 1.83 & $7.30 \mathrm{E}(-4)$ & 1.35 & $1.99 \mathrm{E}(-5)$ & 1.86 \\
$6.25 \times 10^{-3}$ & $2.88 \mathrm{E}(-5)$ & 1.89 & $2.39 \mathrm{E}(-4)$ & 1.61 & $5.32 \mathrm{E}(-6)$ & 1.90 \\
$3.125 \times 10^{-3}$ & $7.53 \mathrm{E}(-6)$ & 1.93 & $6.86 \mathrm{E}(-5)$ & 1.80 & $1.39 \mathrm{E}(-6)$ & 1.94 \\
$1.5625 \times 10^{-3}$ & $1.94 \mathrm{E}(-6)$ & 1.96 & $2.17 \mathrm{E}(-5)$ & 1.66 & $3.54 \mathrm{E}(-7)$ & 1.97 \\
\hline
\end{tabular}


Table 3: Error and convergence rates of Scheme I where $v=v_{r}=0.1$.

\begin{tabular}{||c|c|c|c|c|c|c||}
\hline$\delta t$ & $\|$ error $_{\mathbf{u}} \|_{\infty}$ & Rate & $\|$ error $_{p} \|_{2}$ & Rate & $\|$ error $_{\mathbf{w}} \|_{\infty}$ & Rate \\
\hline $1.25 \times 10^{-2}$ & $1.60 \mathrm{E}(-3)$ & - & $6.90 \mathrm{E}(-3)$ & - & $7.44 \mathrm{E}(-4)$ & - \\
$6.25 \times 10^{-3}$ & $8.24 \mathrm{E}(-4)$ & 0.98 & $3.40 \mathrm{E}(-3)$ & 0.99 & $3.75 \mathrm{E}(-4)$ & 0.99 \\
$3.125 \times 10^{-3}$ & $4.16 \mathrm{E}(-4)$ & 0.99 & $1.70 \mathrm{E}(-3)$ & 1.01 & $1.88 \mathrm{E}(-4)$ & 0.99 \\
$1.5625 \times 10^{-3}$ & $2.09 \mathrm{E}(-4)$ & 0.99 & $8.51 \mathrm{E}(-4)$ & 1.01 & $9.44 \mathrm{E}(-5)$ & 1.00 \\
$7.8125 \times 10^{-4}$ & $1.05 \mathrm{E}(-4)$ & 1.00 & $4.24 \mathrm{E}(-4)$ & 1.00 & $4.73 \mathrm{E}(-5)$ & 1.00 \\
\hline
\end{tabular}

Table 4: Error and convergence rates of Scheme II where $v=v_{r}=0.1$.

\begin{tabular}{||c|c|c|c|c|c|c||}
\hline$\delta t$ & $\|$ error $_{\mathbf{u}} \|_{\infty}$ & Rate & $\left\|_{\text {error }_{p}}\right\|_{2}$ & Rate & $\|$ error $_{\mathbf{w}} \|_{\infty}$ & Rate \\
\hline $2.5 \times 10^{-2}$ & $4.57 \mathrm{E}(-4)$ & - & $3.39 \mathrm{E}(-3)$ & - & $1.55 \mathrm{E}(-5)$ & - \\
$1.25 \times 10^{-2}$ & $1.19 \mathrm{E}(-5)$ & 1.94 & $1.07 \mathrm{E}(-3)$ & 1.66 & $4.19 \mathrm{E}(-6)$ & 1.89 \\
$6.25 \times 10^{-3}$ & $3.03 \mathrm{E}(-5)$ & 1.97 & $2.98 \mathrm{E}(-4)$ & 1.85 & $1.08 \mathrm{E}(-6)$ & 1.96 \\
$3.125 \times 10^{-3}$ & $7.65 \mathrm{E}(-6)$ & 1.98 & $8.49 \mathrm{E}(-5)$ & 1.81 & $2.73 \mathrm{E}(-7)$ & 1.98 \\
$1.5625 \times 10^{-3}$ & $1.93 \mathrm{E}(-6)$ & 1.99 & $3.04 \mathrm{E}(-5)$ & 1.48 & $6.86 \mathrm{E}(-8)$ & 1.99 \\
\hline
\end{tabular}

Table 5: Error and convergence rates of Scheme I where $v=v_{r}=0.01$.

\begin{tabular}{||c|c|c|c|c|c|c||}
\hline$\delta t$ & $\|$ error $_{\mathbf{u}} \|_{\infty}$ & Rate & $\left\|_{\text {error }_{p}}\right\|_{2}$ & Rate & $\|$ error $_{\mathbf{w}} \|_{\infty}$ & Rate \\
\hline $2.5 \times 10^{-2}$ & $2.40 \mathrm{E}(-3)$ & - & $1.27 \mathrm{E}(-2)$ & - & $2.68 \mathrm{E}(-4)$ & - \\
$1.25 \times 10^{-2}$ & $1.20 \mathrm{E}(-3)$ & 1.01 & $6.40 \mathrm{E}(-3)$ & 0.99 & $1.36 \mathrm{E}(-4)$ & 0.97 \\
$6.25 \times 10^{-3}$ & $5.90 \mathrm{E}(-4)$ & 1.00 & $3.20 \mathrm{E}(-3)$ & 1.00 & $6.89 \mathrm{E}(-5)$ & 0.99 \\
$3.125 \times 10^{-3}$ & $2.96 \mathrm{E}(-4)$ & 1.00 & $1.60 \mathrm{E}(-3)$ & 1.00 & $3.46 \mathrm{E}(-5)$ & 0.99 \\
$1.5625 \times 10^{-3}$ & $1.48 \mathrm{E}(-4)$ & 1.00 & $8.03 \mathrm{E}(-4)$ & 1.00 & $1.73 \mathrm{E}(-5)$ & 1.00 \\
\hline
\end{tabular}

Table 6: Error and convergence rates of Scheme II where $v=v_{r}=0.01$.

\begin{tabular}{||c|c|c|c|c|c|c||}
\hline$\delta t$ & $\|$ error $_{\mathbf{u}} \|_{\infty}$ & Rate & $\|$ error $_{p} \|_{2}$ & Rate & $\|$ error $_{\mathbf{w}} \|_{\infty}$ & Rate \\
\hline $5 \times 10^{-2}$ & $1.50 \mathrm{E}(-3)$ & - & $6.90 \mathrm{E}(-3)$ & - & $4.67 \mathrm{E}(-5)$ & - \\
$2.5 \times 10^{-2}$ & $4.06 \mathrm{E}(-4)$ & 1.84 & $2.20 \mathrm{E}(-3)$ & 1.65 & $1.66 \mathrm{E}(-5)$ & 1.49 \\
$1.25 \times 10^{-2}$ & $1.05 \mathrm{E}(-4)$ & 1.95 & $6.19 \mathrm{E}(-4)$ & 1.83 & $4.51 \mathrm{E}(-6)$ & 1.88 \\
$6.25 \times 10^{-3}$ & $2.67 \mathrm{E}(-5)$ & 1.98 & $1.72 \mathrm{E}(-4)$ & 1.85 & $1.16 \mathrm{E}(-6)$ & 1.96 \\
$3.125 \times 10^{-3}$ & $6.74 \mathrm{E}(-6)$ & 1.99 & $5.60 \mathrm{E}(-5)$ & 1.62 & $2.94 \mathrm{E}(-7)$ & 1.98 \\
\hline
\end{tabular}

We take the initial conditions as:

$$
\begin{aligned}
& \left.\mathbf{u}(x, y, t)\right|_{t=0}=\alpha \pi\left(\sin ^{2}(\pi x) \sin (2 \pi y),-\sin (2 \pi x) \sin ^{2}(\pi y)\right)^{T}, \\
& \left.p(x, y, t)\right|_{t=0}=\alpha(\sin (\pi y)-2 / \pi), \\
& \left.\mathbf{w}(x, y, t)\right|_{t=0}=\alpha \pi \sin ^{2}(\pi x) \sin ^{2}(\pi y) .
\end{aligned}
$$


Table 7: Error and convergence rates of Scheme I where $v=v_{r}=0.005$.

\begin{tabular}{||c|c|c|c|c|c|c||}
\hline$\delta t$ & $\|$ error $_{\mathbf{u}} \|_{\infty}$ & Rate & $\|$ error $_{p} \|_{2}$ & Rate & $\|$ error $_{\mathbf{w}} \|_{\infty}$ & Rate \\
\hline $5 \times 10^{-2}$ & $5.40 \mathrm{E}(-3)$ & - & $2.48 \mathrm{E}(-2)$ & - & $3.96 \mathrm{E}(-4)$ & - \\
$2.5 \times 10^{-2}$ & $2.60 \mathrm{E}(-3)$ & 1.06 & $1.27 \mathrm{E}(-2)$ & 0.97 & $2.08 \mathrm{E}(-4)$ & 0.93 \\
$1.25 \times 10^{-2}$ & $1.30 \mathrm{E}(-3)$ & 1.01 & $6.40 \mathrm{E}(-3)$ & 0.99 & $1.06 \mathrm{E}(-4)$ & 0.97 \\
$6.25 \times 10^{-3}$ & $6.46 \mathrm{E}(-4)$ & 1.00 & $3.20 \mathrm{E}(-3)$ & 1.00 & $5.34 \mathrm{E}(-5)$ & 0.99 \\
$3.125 \times 10^{-3}$ & $3.24 \mathrm{E}(-4)$ & 1.00 & $1.60 \mathrm{E}(-3)$ & 1.00 & $2.68 \mathrm{E}(-5)$ & 0.99 \\
\hline
\end{tabular}

Table 8: Error and convergence rates of Scheme II where $v=v_{r}=0.005$.

\begin{tabular}{||c|c|c|c|c|c|c||}
\hline$\delta t$ & $\|$ error $_{\mathbf{u}} \|_{\infty}$ & Rate & $\|$ error $_{p} \|_{2}$ & Rate & $\|$ error $_{\mathbf{w}} \|_{\infty}$ & Rate \\
\hline $5 \times 10^{-2}$ & $1.40 \mathrm{E}(-3)$ & - & $6.34 \mathrm{E}(-3)$ & - & $4.72 \mathrm{E}(-5)$ & - \\
$2.5 \times 10^{-2}$ & $3.83 \mathrm{E}(-4)$ & 1.84 & $1.88 \mathrm{E}(-3)$ & 1.75 & $1.67 \mathrm{E}(-5)$ & 1.50 \\
$1.25 \times 10^{-2}$ & $9.93 \mathrm{E}(-5)$ & 1.95 & $5.18 \mathrm{E}(-4)$ & 1.86 & $4.55 \mathrm{E}(-6)$ & 1.88 \\
$6.25 \times 10^{-3}$ & $2.53 \mathrm{E}(-5)$ & 1.98 & $1.45 \mathrm{E}(-4)$ & 1.84 & $1.17 \mathrm{E}(-6)$ & 1.96 \\
$3.125 \times 10^{-3}$ & $6.37 \mathrm{E}(-6)$ & 1.99 & $4.95 \mathrm{E}(-5)$ & 1.55 & $2.96 \mathrm{E}(-7)$ & 1.98 \\
\hline
\end{tabular}

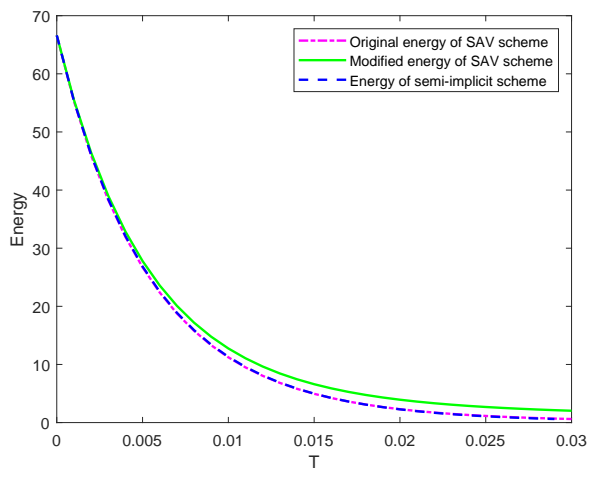

(a) $\Delta t=0.001$

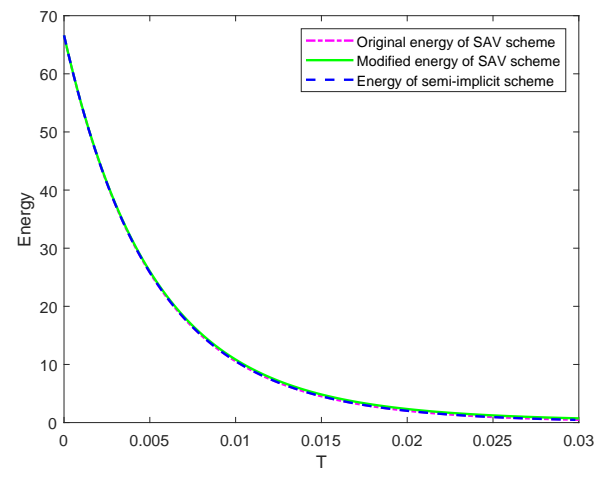

(b) $\Delta t=0.0002$

Figure 1: Comparison of different schemes with $c_{1}=2, \alpha=3, v=v_{r}=1$.

In Figs. 1-4, we compare the time histories of energy computed using the SAV scheme and the usual semi-implicit scheme with $v=v_{r}=1,0.1,0.01$, and 0.005 , respectively. The space is discretized with $128 \times 128$ spectral modes. We observe that at larger time steps in Figs. 3(a) and 4(a), the usual semi-implicit scheme will blow up, but the modified energy of the SAV scheme is still dissipative although the original energy may increase at larger time steps. For sufficiently small time steps, all schemes dissipate the energy at the correct rate. 


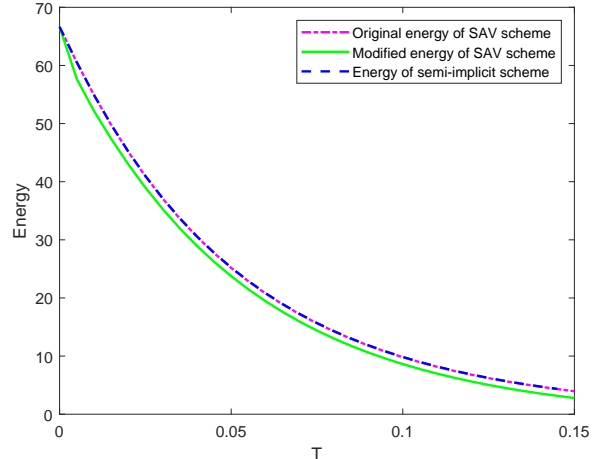

(a) $\Delta t=0.005$

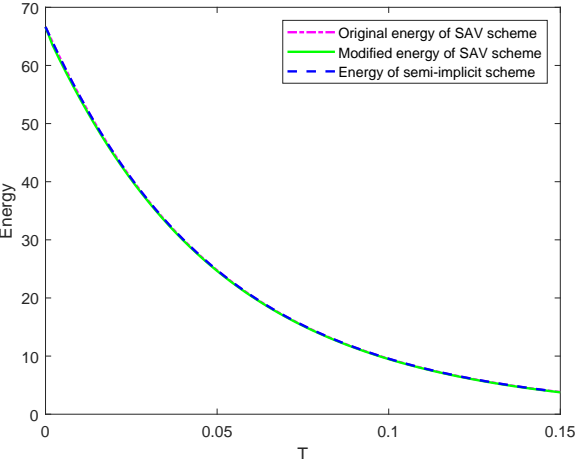

(b) $\Delta t=0.002$

Figure 2: Comparison of different schemes with $c_{1}=2, \alpha=3, v=v_{r}=0.1$.

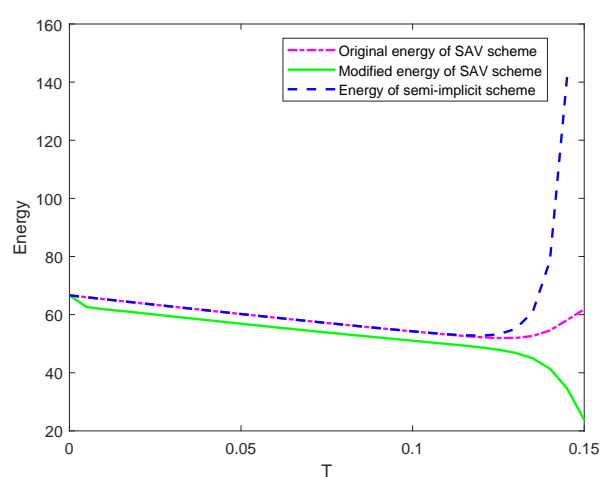

(a) $\Delta t=0.005$

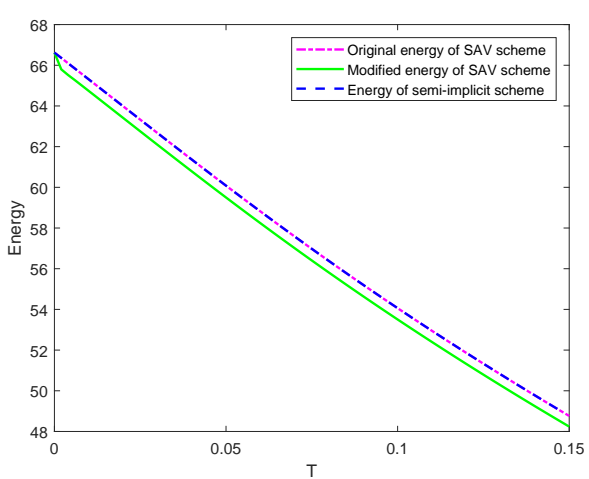

(c) $\Delta t=0.002$

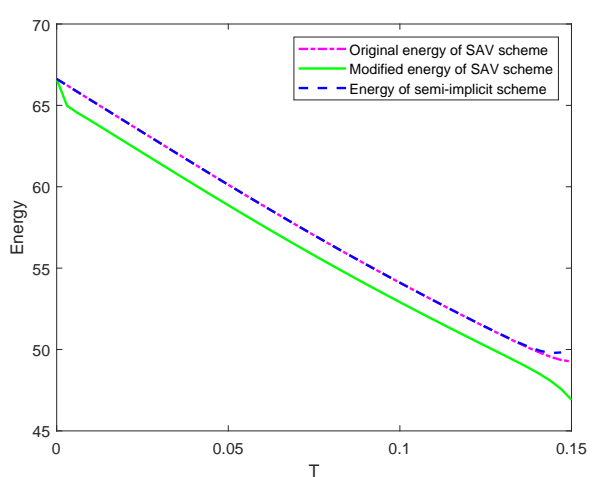

(b) $\Delta t=0.003$

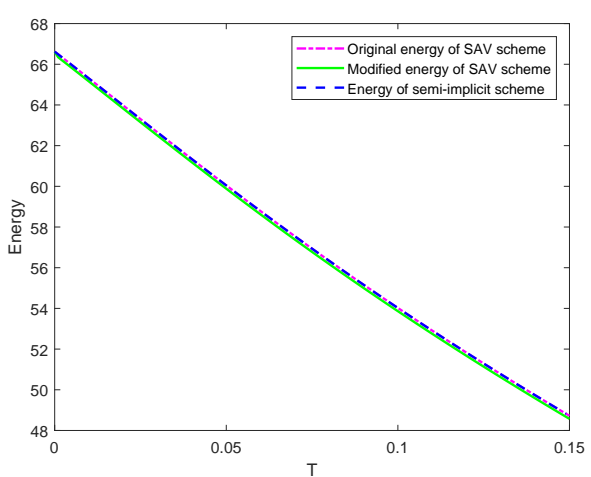

(d) $\Delta t=0.001$

Figure 3: Comparison of different schemes with $c_{1}=2, \alpha=3, v=v_{r}=0.01$. 


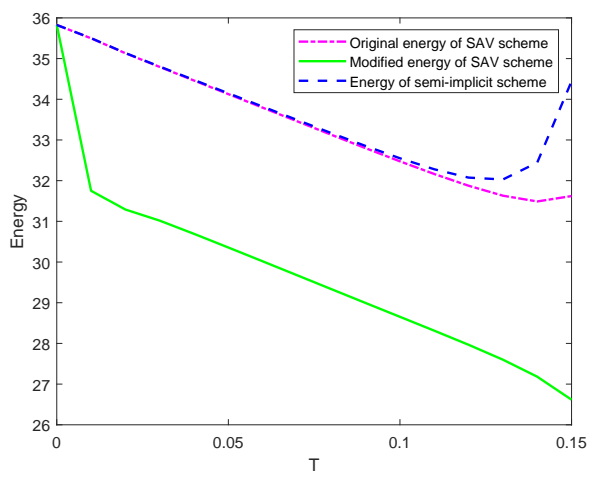

(a) $\Delta t=0.01$

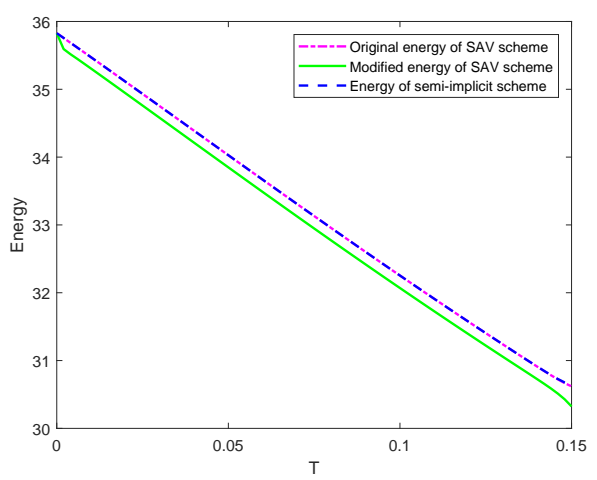

(c) $\Delta t=0.002$

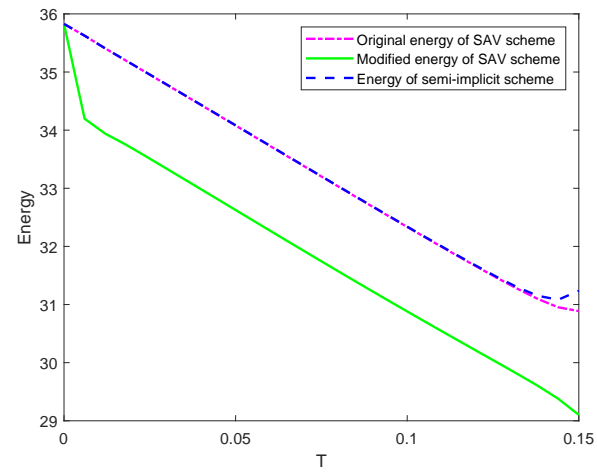

(b) $\Delta t=0.006$

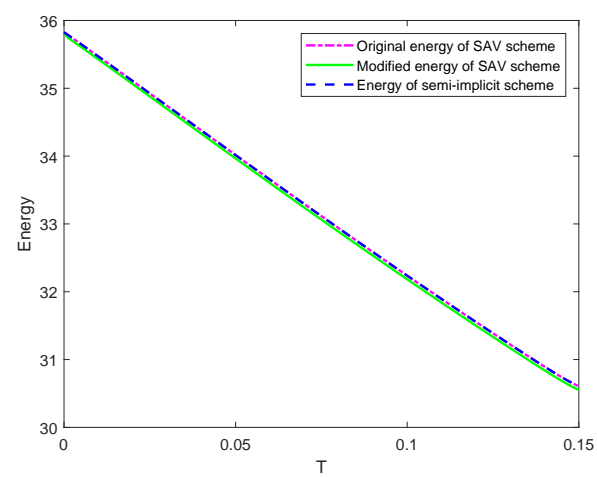

(d) $\Delta t=0.001$

Figure 4: Comparison of different schemes with $c_{1}=2, \alpha=2.2, v=v_{r}=0.005$.

\subsection{A realistic example: Stirring of a passive scalar}

In this example, we set $\mathbf{f}=0, \mathbf{g}=25(x-1), j=1$ and $c_{1}=2$.

To simulate the passive scalar, we supplement the system (5.1) with a passive transport equation [21]

$$
\varphi_{t}+\mathbf{u} \cdot \nabla \varphi=0,
$$

where $\mathbf{u}$ is the velocity in (5.1).

The initial condition for $\varphi$ is defined as:

$$
\left.\varphi\right|_{t=0}= \begin{cases}1, & y<0 \\ 0, & y \geq 0\end{cases}
$$

We first solve the micropolar Navier-Stokes equations (5.1) using the Scheme II with $128 \times 128$ spectral modes in space and with the initial conditions

$$
\left.\mathbf{u}\right|_{t=0}=0,\left.\quad \mathbf{w}\right|_{t=0}=0,\left.\quad p\right|_{t=0}=1 .
$$




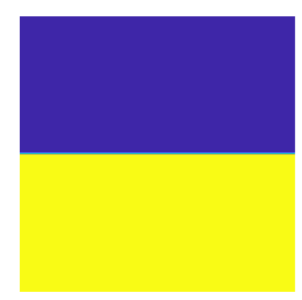

(a) $T=0$

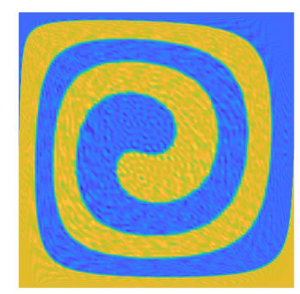

(d) $T=15$

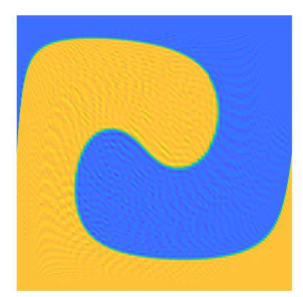

(b) $T=5$

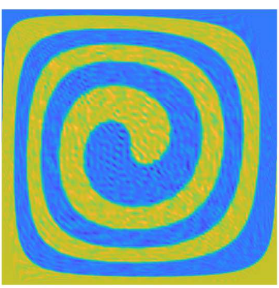

(e) $T=20$

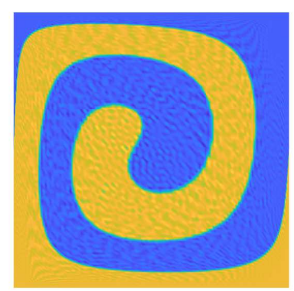

(c) $T=10$

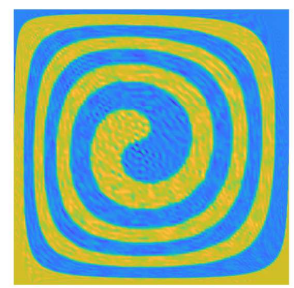

(f) $T=25$

Figure 5: Mixing of a convected passive scalar $\varphi$ with $v=v_{r}=0.1$ and the time step is $\Delta t=0.005$ by means of an applied torque.

Then, we solve the transport equation (5.5) using the fourth-order Runge-Kutta scheme:

$$
\left\{\begin{array}{l}
K_{1}=F\left(\varphi^{n}, t_{n}\right) \\
K_{2}=F\left(\varphi^{n}+\frac{\Delta t}{2} K_{1}, t_{n}+\frac{\Delta t}{2}\right) \\
K_{3}=F\left(\varphi^{n}+\frac{\Delta t}{2} K_{2}, t_{n}+\frac{\Delta t}{2}\right) \\
K_{4}=F\left(\varphi^{n}+\Delta t K_{3}, t_{n+1}\right) \\
\varphi^{n+1}=\varphi^{n}+\frac{\Delta t}{6}\left(K_{1}+2 K_{2}+2 K_{3}+K 4\right) .
\end{array}\right.
$$

where $F(\varphi, t)=-\mathbf{u} \cdot \nabla \varphi$.

Evolutions of the variable $\varphi$ with different values of $v=v_{r}$ are plotted in Figs. 5-7. We observe that as $v$ and $v_{r}$ decrease, the mixing slows down and leads to long waiting times or poor completion of chemical reactions [16].

\section{Concluding remarks}

In this paper, we combined the SAV approach with the pressure-correction method to construct efficient numerical schemes for the micropolar Navier-Stokes equations. The SAV approach allows to treat nonlinear terms explicitly while still being unconditionally 


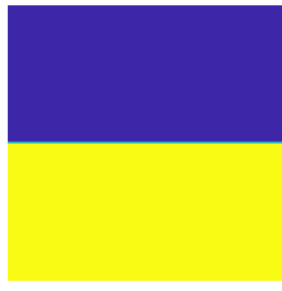

(a) $T=0$

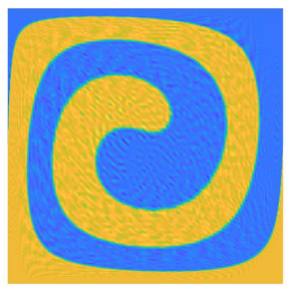

(d) $T=15$

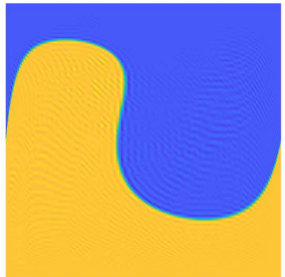

(b) $T=5$

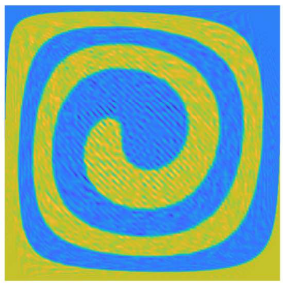

(e) $T=20$

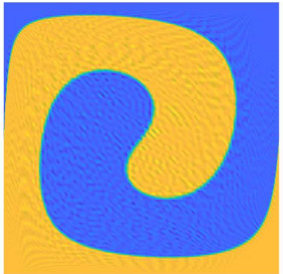

(c) $T=10$

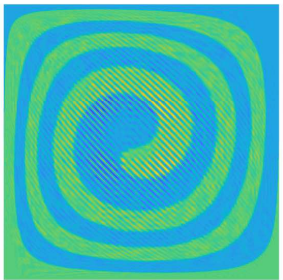

(f) $T=25$

Figure 6: Mixing of a convected passive scalar $\varphi$ with $v=v_{r}=0.01$ and the time step is $\Delta t=0.002$.

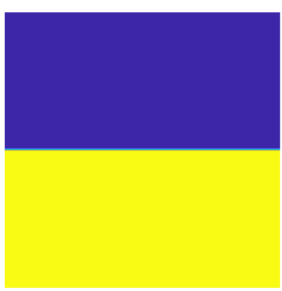

(a) $T=0$

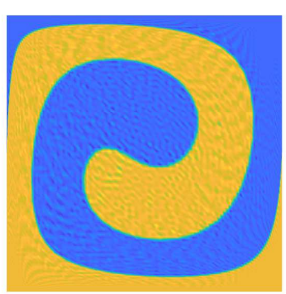

(d) $T=15$

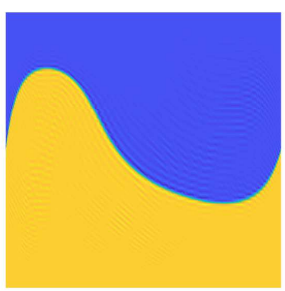

(b) $T=5$

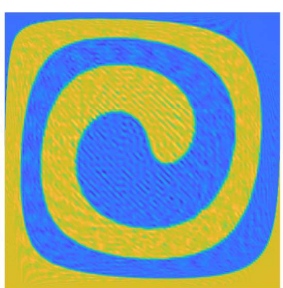

(e) $T=20$

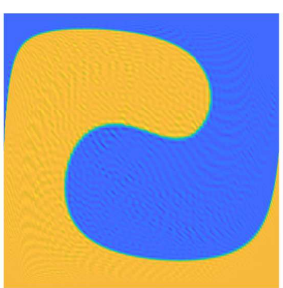

(c) $T=10$

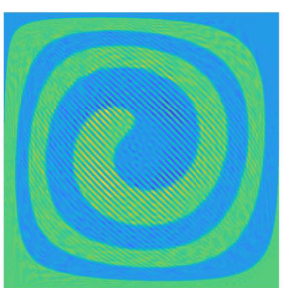

(f) $T=25$

Figure 7: Mixing of a convected passive scalar $\varphi$ with $v=v_{r}=0.005$ and the time step is $\Delta t=0.002$. 
energy stable. The pressure-correction method, standard version is used for constructing the first-order scheme and the rotational version is used to construct second-order scheme, allows us to decouple the pressure from the velocity. Both the semi-discrete first- and second-order schemes enjoy the following remarkable advantages:

- Only a sequence of decoupled linear elliptic equations with constant coefficients need to be solved at each time step so they are very efficient and easy to implement;

- They are unconditionally energy stable with respect to a modified energy.

Unlike in the case of gradient flows for which any consistent Galerkin type spatial discretization will preserve the nice properties of the semi-discrete SAV schemes, special care has to be taken when constructing fully discretization of micropolar Navier-Stokes equations. We constructed a special version of the spectral-Galerkin discretization for the semi-discrete SAV schemes of micropolar Navier-Stokes equations and shown that these fully discrete schemes preserve their essential properties. We presented several numerical experiments to demonstrate the effectiveness of our approach and validate out theoretical results.

We only showed that the SAV schemes are energy stable without error estimates. The main difficulty in carrying out an error analysis is that the nonlinear algebraic equation for $R^{n+1}$ may not have a real positive solution. It is reasonable to expect, and it is true in practice, that for time steps sufficiently small, the nonlinear algebraic equation for $R^{n+1}$ does have a real positive solution, but a rigorous proof is still elusive and will be left as a future endeavor.

\section{Acknowledgments}

The work of J. Shen is supported in part by NSF grant DMS-2012585, and by AFOSR grant FA9550-20-1-0309. The work of N. Zheng is supported in part by CNSF grant 11971407.

\section{References}

[1] E. Ahusborde, M. Azaiez, and J.-P. Caltagirone. A primal formulation for the Helmholtz decomposition. J. Comput. Phys., 225(1):13-19, 2007.

[2] C. Bernardi and Y. Maday. Spectral methods. In Handbook of Numerical Analysis, Vol. V, Handb. Numer. Anal., V, pages 209-485. North-Holland, Amsterdam, 1997.

[3] C. Bernardi, Y. Maday, and F. Rapetti. Discrétisations variationnelles de problèmes aux limites elliptiques, volume 45 of Mathématiques E Applications (Berlin) [Mathematics E Applications]. Springer-Verlag, Berlin, 2004.

[4] F. Chen and J. Shen. Efficient spectral-Galerkin methods for systems of coupled secondorder equations and their applications. J. Comput. Phys., 231(15):5016-5028, 2012.

[5] B.-Q. Dong and Z. Zhang. Global regularity of the 2D micropolar fluid flows with zero angular viscosity. Journal of Differential Equations, 249(1):200-213, 2010. 
[6] A. C. Eringen. Theory of micropolar fluids. Journal of Mathematics and Mechanics, pages 1-18, 1966.

[7] C. Ferrari and R. Gilbert. On lubrication with structured fluids. Applicable Analysis, 15(14):127-146, 1983.

[8] G. P. Galdi and S. Rionero. A note on the existence and uniqueness of solutions of the micropolar fluid equations. International Journal of Engineering Science, 15(2):105-108, 1977.

[9] J. Guermond and J. Shen. On the error estimates for the rotational pressure-correction projection methods. Mathematics of Computation, 73(248):1719-1737.

[10] J.-L. Guermond, P. Minev, and J. Shen. An overview of projection methods for incompressible flows. Computer Methods in Applied Mechanics and Engineering, 195(44-47):6011-6045, 2006.

[11] X. Li, J. Shen, and Z. Liu. New SAV-pressure correction methods for the Navier-Stokes equations: Stability and error analysis. arXiv preprint arXiv:2002.09090, 2020.

[12] L. Lin, Z. Yang, and S. Dong. Numerical approximation of incompressible Navier-Stokes equations based on an auxiliary energy variable. J. Comput. Phys., 388:1-22, 2019.

[13] G. Lukaszewicz. Micropolar Fluids: Theory and Applications. Springer Science \& Business Media, 1999.

[14] G. Łukaszewicz. Long time behavior of 2D micropolar fluid flows. Mathematical and Computer Modelling, 34(5-6):487-509, 2001.

[15] R. H. Nochetto, A. J. Salgado, and I. Tomas. The micropolar Navier-Stokes equations: a priori error analysis. Mathematical Models and Methods in Applied Sciences, 24(07):1237-1264, 2014.

[16] R. H. Nochetto, A. J. Salgado, and I. Tomas. The equations of ferrohydrodynamics: Modeling and numerical methods. Mathematical Models and Methods in Applied Sciences, 26(13):23932449, 2016.

[17] E. Ortega-Torres and M. Rojas-Medar. Optimal error estimate of the penalty finite element method for the micropolar fluid equations. Numerical Functional Analysis and Optimization, 29(5-6):612-637, 2008.

[18] E. E. Ortega-Torres and M. A. Rojas-Medar. Magneto-micropolar fluid motion: Global existence of strong solutions. In Abstract and Applied Analysis, volume 4. Hindawi, 1999.

[19] J.-H. Pyo. Error estimates for the second order semi-discrete stabilized Gauge-Uzawa method for the Navier-Stokes equations. International Journal of Numerical Analysis $\mathcal{E}$ Modeling, 10(1), 2013.

[20] M. A. Rojas-Medar. Magneto-micropolar fluid motion: Existence and uniqueness of strong solution. Mathematische Nachrichten, 188(1):301-319, 1997.

[21] A. J. Salgado. Convergence analysis of fractional time-stepping techniques for incompressible fluids with microstructure. Journal of Scientific Computing, 64(1):216-233, 2015.

[22] V. U. K. Sastry and T. Das. Stability of Couette flow and Dean flow in micropolar fluids. International Journal of Engineering Science, 23(11):1163-1177, 1985.

[23] J. Shen, T. Tang, and L.-L. Wang. Spectral Methods, volume 41 of Springer Series in Computational Mathematics. Springer, Heidelberg, 2011. Algorithms, Analysis and Applications.

[24] J. Shen, J. Xu, and J. Yang. A new class of efficient and robust energy stable schemes for gradient flows. SIAM Rev., 61(3):474-506, 2019. 